\title{
Surface Properties and Corrosion Behavior of Turn-Assisted Deep-Cold-Rolled AISI 4140 Steel
}

\author{
P.R. Prabhu, Deepa Prabhu, Sathyashankara Sharma, and S.M. Kulkarni
}

\author{
(Submitted April 16, 2020; in revised form July 5, 2020; published online August 27, 2020)
}

\begin{abstract}
In this research, the effect of various turn-assisted deep-cold-rolling process parameters on the residual stress, microstructure, surface hardness, surface finish, and corrosion behavior of AISI 4140 steel has been investigated. The examination of the surface morphology of the turned and processed samples was performed by using a scanning electron microscope, energy-dispersive spectroscopy, and atomic force microscopy. Response surface methodology and desirability function approach were used for reducing the number of experiments and finding local optimized conditions for parameters under the study. The results from the residual stress measurements indicate that the rolling force has the highest effect by generating a deeper layer of residual compressive stress. The outcomes of surface hardness and surface finish emphasize that rolling force and number of tool passes are the most significant parameters affecting the responses. Surface studies confirmed the corrosion and its intensity onto the metal surface, and according to atomic force microscopy studies, the surface had become remarkably rough after exposure to the corrosive medium. Improvements in surface microhardness from 225 to $305.8 \mathrm{HV}$, the surface finish from 4.84 down to $0.261 \mu \mathrm{m}$, and corrosion rate from 6.672 down to $3.516 \mathrm{mpy}$ are observed for a specific set of parameters by turn-assisted deep-cold-rolling process. The multiresponse optimization for surface finish and corrosion rate together shows that a ball diameter of $10 \mathrm{~mm}$, a rolling force of $325.75 \mathrm{~N}$, initial roughness of $4.84 \mu \mathrm{m}$, and number of tool passes of 3 give better values for the two responses under consideration with composite desirability of 0.9939 . Based on the experimental work at the optimum parameter setting, the absolute average error between the experimental and predicted values for the corrosion rate is calculated as $3.2 \%$.
\end{abstract}

Keywords corrosion rate, deep cold rolling, residual stress, scanning electron microscope, surface hardness, surface finish

\section{Introduction}

Most of the components used in the aerospace and automobile industry require excellent surface characteristics as they work under severe conditions (Ref 1). Fatigue is one of the main reasons for the failure of these components. About $90 \%$ of all mechanical service failures are due to fatigue (Ref 2 , 3 ). The fatigue failure relies entirely upon the ultimate strength or yield stress of the material. But actuality, different factors, for example, geometry, quality of the surface, type of material, residual stresses, loading direction, size of the grain, surrounding atmosphere, operating temperature may reduce the service life of a component (Ref 4, 5). Fatigue occurs due to cracks originated on free surfaces, and therefore fatigue life is reliant on the surface finish (Ref 6-8). Residual compressive stress on

P.R. Prabhu and Sathyashankara Sharma, Department of Mechanical and Manufacturing Engineering, Manipal Institute of Technology, Manipal Academy of Higher Education, Manipal, Karnataka 576104, India; Deepa Prabhu, Department of Chemistry, International Center for Applied Sciences, Manipal Academy of Higher Education, Manipal, Karnataka 576104, India; S.M. Kulkarni, Department of Mechanical Engineering, National Institute of Technology, Surathkal, Karnataka 575025, India. Contact e-mail: deepa.prabhu@manipal.edu.

the surface and near surface of a part is beneficial as it enhances the fatigue life by slowing down the rate of crack propagation (Ref 9, 10). Tensile residual stress on the surface of the component is generally detrimental as it decreases the fatigue life by speeding the rate of crack propagation and lowering the resistance to environmentally assisted cracking (Ref 11,12 ). Generally, turning results in tensile residual stress, which is not beneficial for fatigue life. Hence, secondary processes are adopted to improve fatigue life. In addition to the failure due to fatigue, corrosion of machine components results in significant financial losses and may also be responsible for acute and costly breakdowns (Ref 13). Due to the enormous loss, the problems related to corrosion need to be considered seriously. Effective protective techniques need to be applied to reduce corrosion. Some of the methods to prevent corrosion are using metals and alloys having excellent resistance to corrosion, protective coating, passive layers, surface finishing operations, etc.

The corrosion of high-strength steel and its association with surface morphology have been a concern for product sustainability. This is due to the layer of chromium oxide on the metal surface preventing corrosion. The data related to the association between the surface morphology and corrosion behavior of stainless steel can be found in the literature (Ref 14). The surface morphology of a metal constituent is determined by surface finishing processes that affect the corrosion. In specific, the surfaces prepared by mechanical processes are greatly affected by the wear processes. Different surface textures are obtained with different wear modes resulting in different surface finishes. To explain the exclusive features of the surfaces, several roughness parameters have been established, and among them, the root-mean-square average roughness $\left(R_{\mathrm{q}}\right)$ 
and the average surface roughness $\left(R_{\mathrm{a}}\right)$ are the most commonly used parameters (Ref 14).

Components used in the present day ask for materials with superior surface characteristics such as high resistance to corrosion and wear, improved surface hardness and finish, residual stress (Ref 15). Using alloys will increase the cost of the components; hence, few mechanical surface enhancement (MSE) methods are utilized. The MSE methods such as shot peening, laser shock peening, burnishing will impart the compressive residual stress near the surface and will enhance the surface properties such as wear resistance, residual compressive stress, corrosion resistance, surface hardness and finish (Ref 11, 12, 16-19). However, the methods revealed above, except burnishing, always produce rough surface and would remove the advantageous impacts of a surface nanocrystalline microstructure (Ref 15). Enormous roughness may promote stress concentration at specific sites and hence initiate crack, particularly under fatigue loading conditions (Ref 20). Hence, decreasing the surface roughness is as important as surface nanocrystallization on improving the material's performance, including corrosion resistance (Ref 21). One of the MSE methods, deep cold rolling (DCR), is getting attention nowadays as it is a fast, chipless, and economical surface enhancement method applicable to prevailing conventional or computer numerical control (CNC) machine tools. Further producing a deep layer of compression and nanocrystalline microstructure on the surface of samples, it also can lessen the surface roughness of materials and enhance the fatigue life of engineering components without affecting their bulk properties (Ref 22-34). There are numerous parameters to be considered, and the effects of each of these parameters are dependent on other variables. In addition to the experimental studies, predictive models are necessary to understand further the mechanisms that drive the surface integrity changes and to discover the optimum machining settings that would lead to desirable surface integrity (Ref 35 ). So, the design of experiments (DOE) is utilized where the effect of various process parameters can be studied simultaneously with these proficient structures, rather than in a trial-and-error approach, giving truly reproducible outcomes (Ref 26, 36-39). Response surface methodology (RSM) is a mixture of numerical and measurable strategies for the experimental model structure. Via a cautious plan of trials, the goal is to optimize a response/s, which is affected by various factors (Ref 40-43).

AISI 4140 steel possesses excellent mechanical properties and is often used as axle/crankshaft material in automobile industries. Cracking of this part will seriously affect the safety of the vehicles. Y.X. Zhao et al. (Ref 44) studied short fatigue crack propagation and found that effective microcracks initiated from the ferrite on the surfaces of the material. Therefore, improving the surface properties of machine components that work under fatigue and corrosion is an effective way to enhance the service life of the components. Although surface alteration by application of the deep-cold-rolling process is quite new, it has received substantial demand due to enhanced residual compressive stress (Ref 29, 45), surface work hardening (Ref $28,46)$, surface finish (Ref 18,47$)$ and grain refinement which in turn will have the positive effect on corrosion properties. Therefore, a surface enhancement method improving the surface properties after the machining would be beneficial along with the effective use of the design of experiment to reduce the time and number of experiments to be conducted. Based on the above literature analysis, it is clear that the effects of deep-cold-rolling process parameters on the surface integrity of titanium alloys, magnesium alloys, and hardened steel have been explored (Ref 4, 23, 28, 34, 45, 48, 49). However, the literature on the effects of various deep rolling parameters on the surface integrity of AISI 4140 steel is limited (Ref 49, 50). A survey in the literature also indicates that there are few investigations about the application of DOE methods for optimizing the process parameters for enhanced surface characteristics (Ref 4, 51).

Therefore, this study has been focused on achieving the enhanced surface properties (residual stress, surface hardness, surface finish, and corrosion behavior) of AISI 4140 steel through a custom-made turn-assisted deep-cold-rolling (TADCR) tool and setup and optimizing the various process parameters (ball diameter, rolling force, initial roughness of the workpiece, and number of tool passes) for better responses by using response surface methodology and desirability function approach. The corrosion behavior of AISI 4140 steel for the turned and turn-assisted deep-cold-rolled samples was made to undergo a weight loss (gravimetric analysis) technique for corrosion rate measurement in $3.5 \% \mathrm{NaCl}$ solution. Scanning electron microscope (SEM) and energy-dispersive spectroscopy (EDS) analyses were used for surface morphology studies and elemental analysis of the surface layers, respectively. The atomic force microscopy (AFM) analysis was carried out to reveal the relation between corrosion rate and average surface roughness $\left(R_{a}\right)$.

\section{Material and Experimental Details}

\subsection{Material and Experimental Setup}

The study conducted on AISI 4140 steel contains nickel, chromium, and molybdenum, which increases its passivation and consequently enhances resistance to corrosion. Thus, this is a suitable steel to be used in an aggressive medium. It finds vast application in automobile and aerospace industry for the manufacture of various parts experiencing fatigue loads (Ref $52,53)$. The primary chemical ingredients of the material used in $\mathrm{wt} \%$ are as follows: $\mathrm{C}-0.40, \mathrm{Si}-0.27, \mathrm{Mn}-0.66, \mathrm{Cr}-1.20$, $\mathrm{Mo}-0.25, \mathrm{Ni}-0.16, \mathrm{Cu}-0.12$. The mechanical properties of the as-bought material are as follows: yield strength-850 $\mathrm{MPa}$, ultimate tensile strength-949 MPa, Vickers hardness-225 VHN. Specimens are first turned to the required diameter to render similar surface roughness. The detailed view of the experimental setup retrofitted on the lathe is given in Fig. 1. Four-component Kistler dynamometer of type 9272A was used for the measurement of forces while TADCR is attached on the cross slide of the lathe. The force values were recorded in a laptop through DynoWare force measuring software. To restrict the bending of the specimen at a higher rolling force, a follower rest/backrest mechanism was provided. Here, the sample will have support from the back as well as from the top with rolling contact elements as shown in Fig. 1 to reduce bending of the specimen and friction. To ensure a point of contact between the follower rest and the specimen, tungsten carbide balls were provided.

\subsection{CCD Experiments}

Four process factors as shown in Table 1 are identified to have a significant effect on the TADCR process. These process 

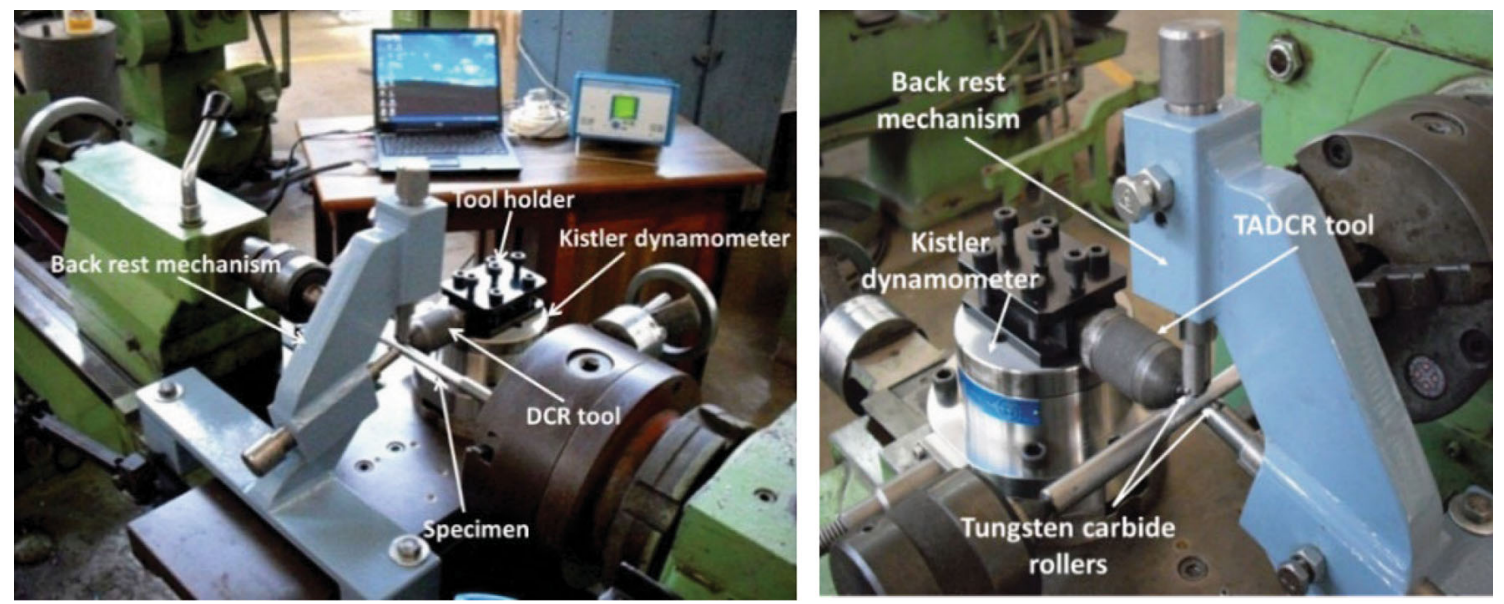

Fig. 1 Photographic view of the experimental setup with backrest and rolling contact elements

Table 1 Variables and levels for detailed CCD experiments

\begin{tabular}{lllll}
\hline & & \multicolumn{2}{c}{ Levels } \\
\cline { 3 - 5 } Symbol & \multicolumn{1}{c}{ Factors } & Low & Medium & High \\
\hline$X_{1}$ & Ball diameter, $m$ m & 6 & 8 & 10 \\
$X_{2}$ & Rolling force, $N$ & 250 & 500 & 750 \\
$X_{3}$ & Initial $R_{\mathrm{a}}$ of the workpiece, $\mu \mathrm{m}$ & 4.84 & 6.15 & 7.46 \\
$X_{4}$ & Number of passes & 1 & 2 & 3 \\
\hline
\end{tabular}

Table 2 Residual stress results of turned and TADCR samples at a specific condition

\begin{tabular}{lc}
\hline Sample & Residual stress, MPa \\
\hline Turned & 93.83 \\
TADCR at $250 \mathrm{~N}$ & -292.93 \\
TADCR at $750 \mathrm{~N}$ & -568.74 \\
\hline
\end{tabular}

parameters are chosen at three levels. During the experiments, apart from these parameters, the remaining three parameters are set at favorable values identified from screening experiments (i.e., ball material - tungsten carbide, feed rate $-36 \mathrm{~mm} / \mathrm{min}$ and lubricant oil-brake oil). The scheme of central composite design (CCD) adopted with three levels of each parameter is shown in Table 1.

\subsection{Response Characterization Methods}

Residual stresses (RS) imparted due to TADCR are measured using Rigaku X-ray residual stress analyzer. Residual stress was measured at different depths of $0.05,0.1,0.2,0.3$, 0.4 , and $0.5 \mathrm{~mm}$ from the workpiece surface. Surface hardness (SH) of samples was measured with the help of Matsuzawa Micro Vickers hardness tester. The Vickers indentor loaded with $4.905 \mathrm{~N}$ for a loading period of $10 \mathrm{~s}$ is used to evaluate microhardness variations with increments of $25 \mu \mathrm{m}$ between two successive indentations. The surface of the specimen was examined using different characterization techniques. Microstructure examination of the test surface was done before and after the TADCR process by utilizing optical microscopy.

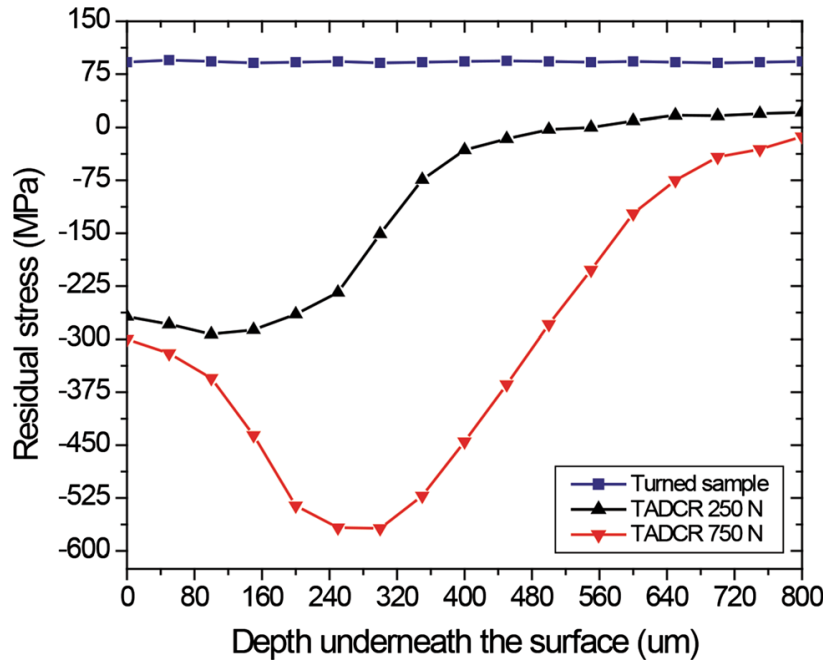

Fig. 2 Depth profiles of residual stress for the untreated and treated specimens

A Taylor Hobson Surtronic 3+ surface measuring instrument was used to discover the surface structure and surface finish at a microscale. An IB342 Innova model atomic force microscopy (AFM) was utilized to measure the surface finish and surface structure in nanoscale. The corrosion rate (CR) of the samples before and after TADCR at a various combination of parameters is calculated by using the weight loss (WL) method. The surface is checked through a JEOL JSM-6380L analytical scanning electron microscope (SEM) connected with the 


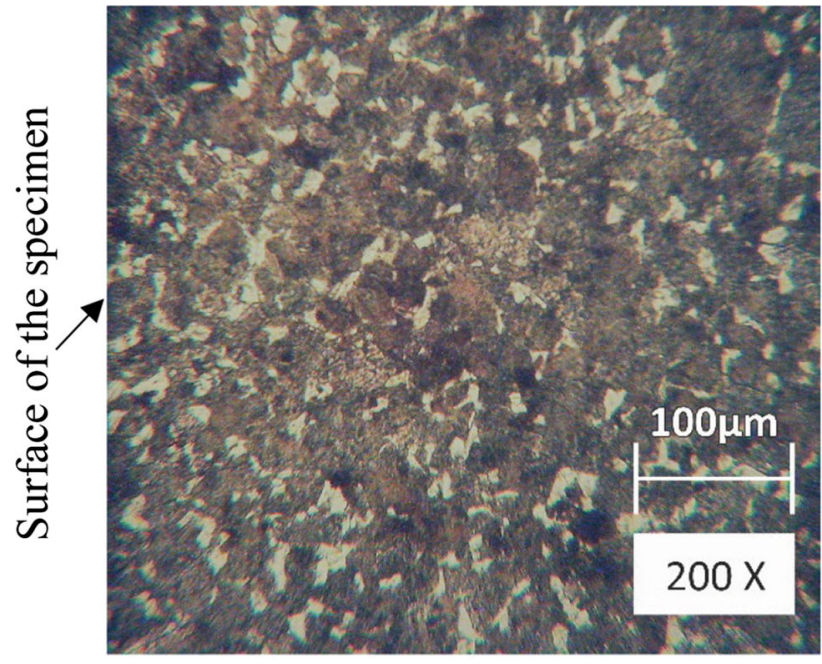

(a)

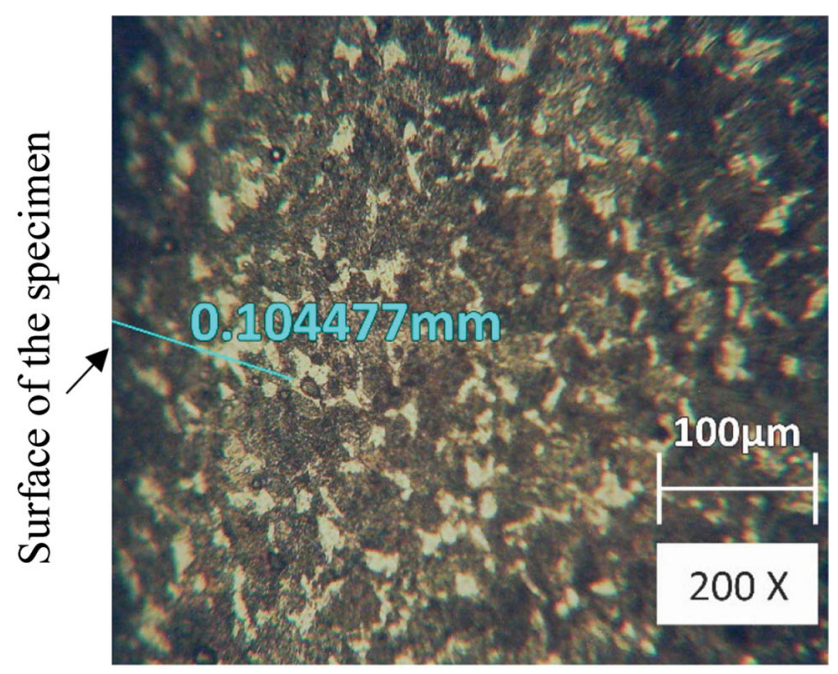

(b)

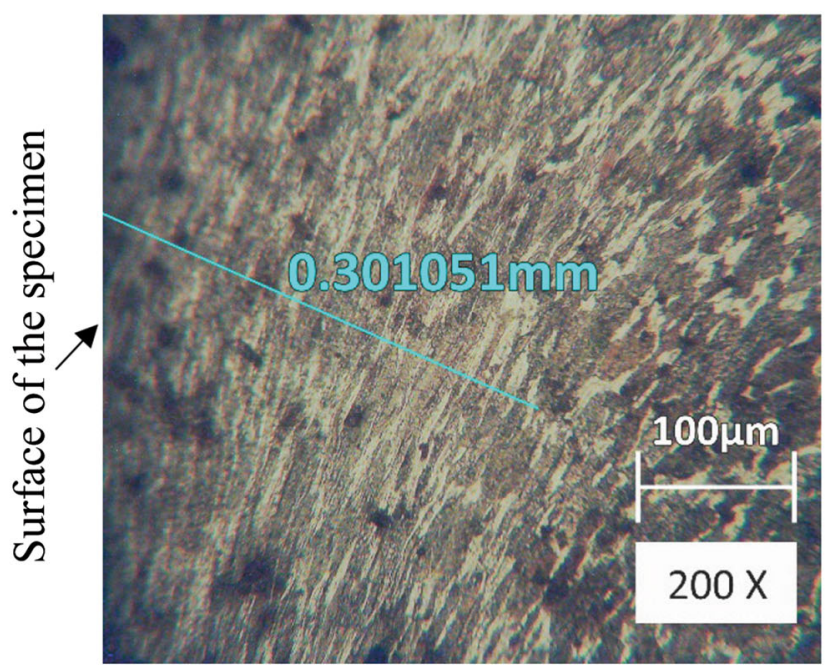

(c)

Fig. 3 Cross-sectional micrographs displaying near-surface microstructures in (a) turned and (b), (c) TADCR samples with 250 and $750 \mathrm{~N}$ force

energy-dispersive $\mathrm{x}$-ray (EDX) to examine the surface morphology and to evaluate the elements on the surface of the specimen before and after immersing in the corrosive medium.

\subsection{Corrosion Investigations using the Weight Loss Method}

The weight loss experiments were performed on cylindrical specimens having the dimensions $9.48 \mathrm{~mm}$ diameter and $10 \mathrm{~mm}$ length. Turned and deep-cold-rolled specimens at various parameter settings were dipped in $100-\mathrm{mL}$ glass beakers comprising $60 \mathrm{~mL} 3.5 \% \mathrm{NaCl}$ solution for immersion time of $936 \mathrm{~h}$ at room temperature $\left(30 \pm 1{ }^{\circ} \mathrm{C}\right)$. To identify the specimens dipped in the corrosive medium, numbered labels were pasted on each beaker. Before taking all the readings, the surface exposed to the corrosive medium is thoroughly washed with double distilled water, degreased and dried with acetone. Before measurement, the samples were kept in a desiccator over a silica gel for $24 \mathrm{~h}$. The corrosion product layer is scratched off with a fiber brush and promptly drenched in the pickling arrangement containing concentrated nitric acid $(86 \%)$ for 5 min (ASTM G1). Once all the corrosion products were completely removed from the surface, the samples were flushed with deionized water and ethanol, dried with a surge of air, and weighed again to decide weight reduction. Duplicate tests were conducted for all the weight loss tests. The variance between the initial and final weights of the sample is used to determine the CR. The corrosion rate (in mpy) is calculated by using the following formula (54): $\mathrm{CR}=\frac{\mathrm{KW}}{\mathrm{ATD}}$, where $K$ is a constant $\left(3.45 \times 10^{6}\right), W$ is the difference between the initial and final weights of the sample in gms, $D$ is the density of the sample in $\mathrm{g} / \mathrm{cm}^{3}, A$ is the area of the sample exposed to corrosive medium in $\mathrm{cm}^{2}$, and $T$ is the immersion time in hours.

\section{Results and Discussion}

\subsection{Residual Stress Analysis}

Residual stress in specimens treated with the TADCR process is given in Table 2. The compressive residual stress of TADCR specimens is far higher when compared with the untreated/turned 
specimen. This increase is because of the cold working proven as elongation of grains. Figure 2 shows a considerable amount of residual compressive stresses at the surface and near-surface area after the TADCR process. Maximum residual compressive stress measured experimentally is $569 \mathrm{MPa}$ immediately below the surface rolled at $750 \mathrm{~N}$ force. This is significantly more compared to residual stress in the turned sample measured at $+94 \mathrm{MPa}$ (tensile). The maximum residual compressive stress of $293 \mathrm{MPa}$ is seen in the sample deep cold rolled at a lower force of $250 \mathrm{~N}$. From Fig. 2, it is also noticed that the penetration of compressive residual stress layer for TADCR with $750 \mathrm{~N}$ is $0.3 \mathrm{~mm}$ and $250 \mathrm{~N}$ rolling force is $0.1 \mathrm{~mm}$. Thus, $750 \mathrm{~N}$ rolling force induces the compression layer to a depth nearly three times greater than at $250 \mathrm{~N}$ rolling force. This could be seen manifested as grain elongation in microstructure, as explained below.

The microstructure of the unrolled (as-turned) specimen shown in Fig. 3a shows equiaxial proeutectoid ferrite grains with pearlitic phases. The white patches show the proeutectoid ferritic phases with large colonies of pearlitic (light area) regions. The microstructure remains the same from the surface toward the core of the specimen. The microstructure of the TADCR specimen operated with $750 \mathrm{~N}$ rolling force displays elongated grains, as shown in Fig. 3c. Here, the deformation and elongation of grain in the direction of rolling can be seen. The intensity of elongation is more near the surface where the tool is in contact with the workpiece, and also, deeper penetration is observed. As the depth increases, the grain elongation intensity reduces. The farthest region from the surface seems to be unaffected by the deep-coldrolling process. Figure $3 \mathrm{~b}$ shows the microstructure of the TADCR specimen, where the process was done with the rolling force of $250 \mathrm{~N}$, which is much less than the previous case. From Fig. $3 b$, it is seen that the grains are partially elongated to a depth of around $0.1 \mathrm{~mm}$ from the surface. The reason is that the force applied on the workpiece surface is mostly utilized to level the peaks and valleys. Thus, the generated residual compressive stress is very less as compared to the previous condition. From this, we can conclude that the rolling force has a significant effect on the generation of residual compressive stress on the surface. The higher the rolling force, the deeper will be the layer of residual compressive stress generated. Similar results can also be seen from the residual stress analysis shown in Fig. 2.

\subsection{Surface Hardness}

The average values of surface hardness with different process parameters are recorded in Table 3. From the analysis, the rolling force has the highest effect on the surface hardness, followed by ball diameter with a contribution of 66.22 and $16.29 \%$, respectively. Hardness at the surface and near surface increases with an increase in ball diameter, rolling force, and the number of passes and decreases with an increase in the initial roughness of the workpiece.

The subsurface microhardness found at various depths of the specimen is shown in Fig. 4. The nature of the curve obtained for microhardness of TADCR samples concerning the depth at different rolling forces is almost similar. The highest hardness is observed at the surface, then gradually decreases, and reaches the value of the turned sample. The average microhardness of the turned specimen on the surface is around $225 \mathrm{HV}$. Maximum hardness of about $306 \mathrm{HV}$ is recorded on the surface for TADCR with a force of $750 \mathrm{~N}$, and the hardness is found to decrease when we go deep from the surface and ultimately settle down at hardness of turned sample at a depth
Table 3 Scheme of central composite experimental design and measured values of corrosion rate, surface hardness, and surface finish

\begin{tabular}{|c|c|c|c|c|c|c|c|}
\hline \multicolumn{5}{|c|}{ Central composite design } & \multirow[b]{2}{*}{ CR, mpy } & \multirow[b]{2}{*}{ SH, VHN } & \multirow[b]{2}{*}{$\mathrm{SF}, \boldsymbol{\mu m}$} \\
\hline Run order & $X_{1}$ & $X_{2}$ & $X_{3}$ & $X_{4}$ & & & \\
\hline 1 & 8 & 500 & 7.46 & 2 & 4.383 & 258.6 & 0.411 \\
\hline 2 & 10 & 250 & 4.84 & 3 & 3.516 & 263.6 & 0.269 \\
\hline 3 & 8 & 500 & 6.15 & 3 & 4.462 & 265.4 & 0.339 \\
\hline 4 & 8 & 500 & 4.84 & 2 & 4.351 & 265.9 & 0.392 \\
\hline 5 & 6 & 250 & 7.46 & 1 & 4.635 & 241.5 & 0.618 \\
\hline 6 & 6 & 750 & 4.84 & 1 & 4.887 & 270.6 & 0.569 \\
\hline 7 & 10 & 250 & 7.46 & 1 & 4.446 & 247.3 & 0.494 \\
\hline 8 & 10 & 750 & 4.84 & 1 & 4.146 & 297.8 & 0.466 \\
\hline 9 & 8 & 500 & 6.15 & 2 & 4.619 & 262.4 & 0.399 \\
\hline 10 & 6 & 750 & 7.46 & 3 & 4.013 & 265.4 & 0.326 \\
\hline 11 & 6 & 750 & 7.46 & 1 & 4.210 & 264.4 & 0.584 \\
\hline 12 & 10 & 750 & 4.84 & 3 & 4.083 & 305.8 & 0.261 \\
\hline 13 & 8 & 500 & 6.15 & 2 & 4.619 & 263.6 & 0.400 \\
\hline 14 & 6 & 250 & 7.46 & 3 & 4.270 & 245.7 & 0.373 \\
\hline 15 & 8 & 250 & 6.15 & 2 & 4.651 & 247.7 & 0.486 \\
\hline 16 & 6 & 750 & 4.84 & 3 & 4.794 & 275.4 & 0.335 \\
\hline 17 & 6 & 250 & 4.84 & 1 & 4.225 & 243.7 & 0.69 \\
\hline 18 & 8 & 500 & 6.15 & 2 & 4.619 & 262.4 & 0.399 \\
\hline 19 & 8 & 500 & 6.15 & 2 & 4.619 & 263.6 & 0.399 \\
\hline 20 & 10 & 750 & 7.46 & 3 & 3.800 & 274.3 & 0.371 \\
\hline 21 & 10 & 750 & 7.46 & 1 & 4.083 & 285.4 & 0.596 \\
\hline 22 & 10 & 500 & 6.15 & 2 & 4.003 & 269.3 & 0.391 \\
\hline 23 & 10 & 250 & 4.84 & 1 & 3.516 & 255 & 0.451 \\
\hline 24 & 8 & 500 & 6.15 & 2 & 4.619 & 263.6 & 0.394 \\
\hline 25 & 8 & 500 & 6.15 & 2 & 4.619 & 263.6 & 0.398 \\
\hline 26 & 8 & 750 & 6.15 & 2 & 4.719 & 283.4 & 0.391 \\
\hline 27 & 6 & 250 & 4.84 & 3 & 4.385 & 250.3 & 0.428 \\
\hline 28 & 8 & 500 & 6.15 & 2 & 4.619 & 263.6 & 0.398 \\
\hline 29 & 10 & 250 & 7.46 & 3 & 4.115 & 250.3 & 0.328 \\
\hline 30 & 8 & 500 & 6.15 & 1 & 4.609 & 258.2 & 0.503 \\
\hline 31 & 6 & 500 & 6.15 & 2 & 4.397 & 254.2 & 0.460 \\
\hline
\end{tabular}

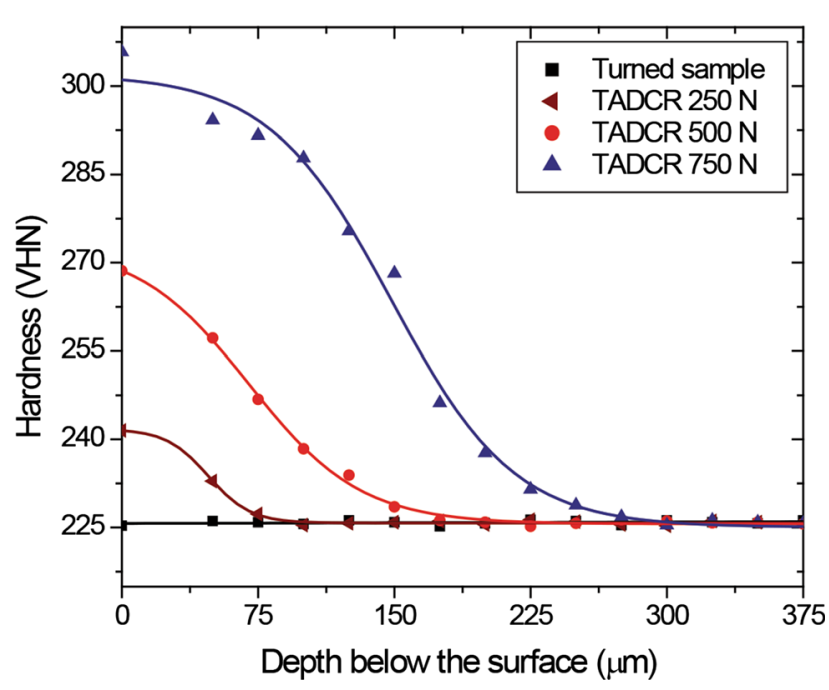

Fig. 4 Plot showing subsurface microhardness found at various depths of the specimen [10 $\mathrm{mm}, 4.84 \mu \mathrm{m}, 3]$

of about $300 \mu \mathrm{m}$. It could also be observed that surface microhardness settles at a depth of 175 and $100 \mu \mathrm{m}$ for TADCR with 500 and $250 \mathrm{~N}$ force, respectively. This variation 


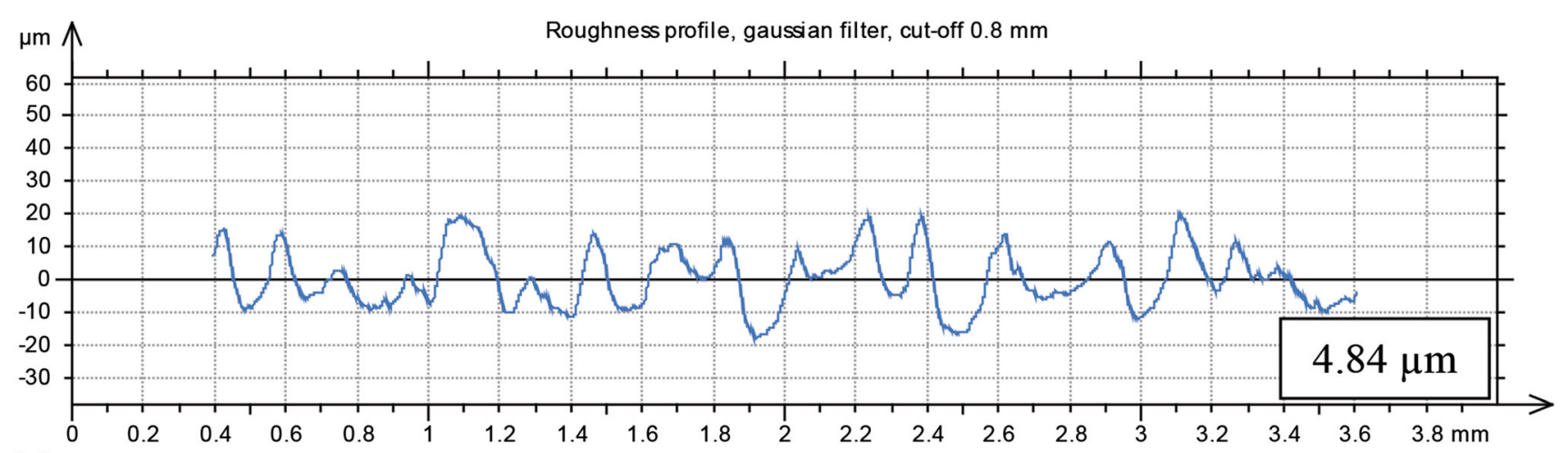

(a)

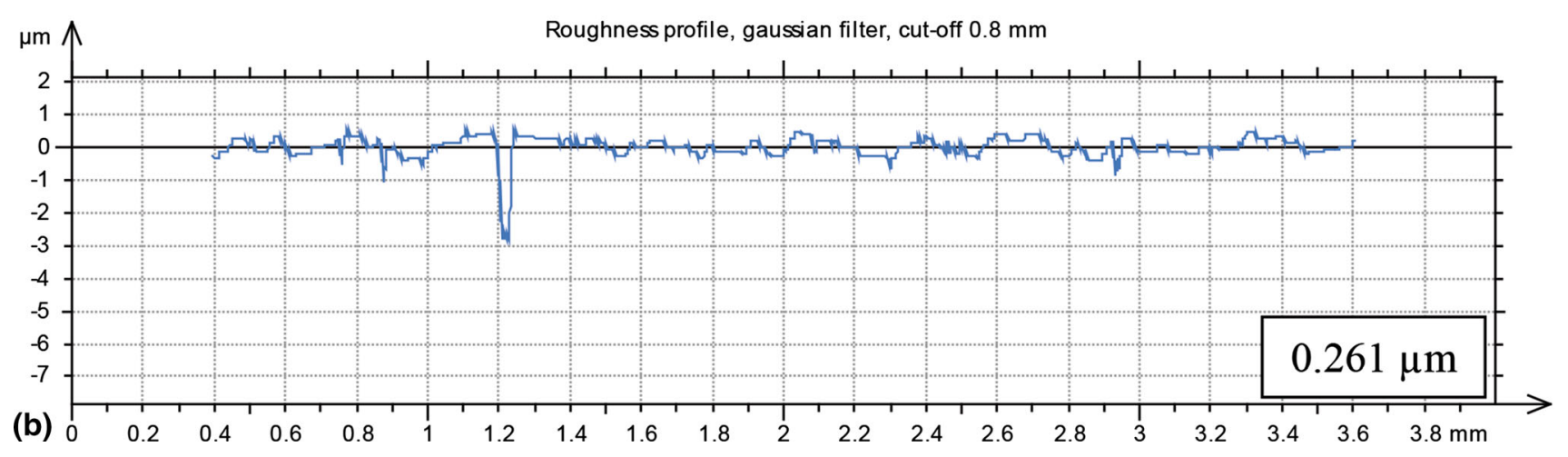

Fig. 5 Surface profiles of the (a) turned and (b) TADCR specimen at $750 \mathrm{~N}$ force

Table 4 ANOVA table for corrosion rate

\begin{tabular}{|c|c|c|c|c|c|c|}
\hline Source & DF & Adj SS & Adj MS & $F$-Value & $P$-Value & PC, \% \\
\hline$X_{1}$ & 1 & 0.93804 & 0.938041 & 300.30 & 0.000 & 33.03 \\
\hline$X_{2}$ & 1 & 0.05303 & 0.053033 & 16.98 & 0.001 & 1.87 \\
\hline$X_{3}$ & 1 & 0.00014 & 0.000140 & 0.04 & 0.835 & 0.00 \\
\hline$X_{4}$ & 1 & 0.09675 & 0.096746 & 30.97 & 0.000 & 3.41 \\
\hline$X_{1} X_{2}$ & 1 & 0.00109 & 0.001089 & 0.35 & 0.563 & 0.04 \\
\hline$X_{1} X_{3}$ & 1 & 0.34406 & 0.344060 & 110.15 & 0.000 & 12.12 \\
\hline$X_{1} X_{4}$ & 1 & 0.00211 & 0.002105 & 0.67 & 0.424 & 0.07 \\
\hline$X_{2} X_{3}$ & 1 & 0.82346 & 0.823464 & 263.62 & 0.000 & 29.00 \\
\hline$X_{2} X_{4}$ & 1 & 0.00063 & 0.000631 & 0.20 & 0.659 & 0.02 \\
\hline$X_{3} X_{4}$ & 1 & 0.08696 & 0.086964 & 27.84 & 0.000 & 3.06 \\
\hline$X_{1}^{2}$ & 1 & 0.31012 & 0.310122 & 99.28 & 0.000 & 10.92 \\
\hline$X_{2}^{2}$ & 1 & 0.05050 & 0.050500 & 16.17 & 0.001 & 1.78 \\
\hline$X_{3}^{2}$ & 1 & 0.08271 & 0.082710 & 26.48 & 0.000 & 2.91 \\
\hline$X_{4}^{2}$ & 1 & 0.00027 & 0.000274 & 0.09 & 0.771 & 0.01 \\
\hline Error & 16 & 0.04998 & 0.003124 & & & 1.76 \\
\hline Total & 30 & 2.83985 & & & & \\
\hline \multicolumn{7}{|c|}{${ }^{*} \mathrm{PC}$ - percentage contribution } \\
\hline
\end{tabular}

complements the variation in residual stress shown in Fig. 2. Higher hardness at the surface logically diminishing is because of the differential measure of cold work experienced by the material.

\subsection{Surface Finish}

Surface finish values after TADCR with various factors are recorded and given in Table 3. The results indicate that the number of tool passes and ball diameter are the two most significant factors affecting the surface finish with 68.22 and
$10.35 \%$ contributions, respectively. The surface finish increases with the increase in the ball diameter and number of tool passes and decreases with the increase in rolling force and initial roughness of the workpiece. One of the roughness profiles of the turned specimen is shown in Fig. 5a. A higher value of $R_{a}$ is observed because the deviation from the mean line is significant. Similarly, Fig. 5b shows the roughness profile of the TADCR sample, where we have observed lower $R_{a}$ value (the deviation from the mean line is small), suggesting improvement in surface finish. 


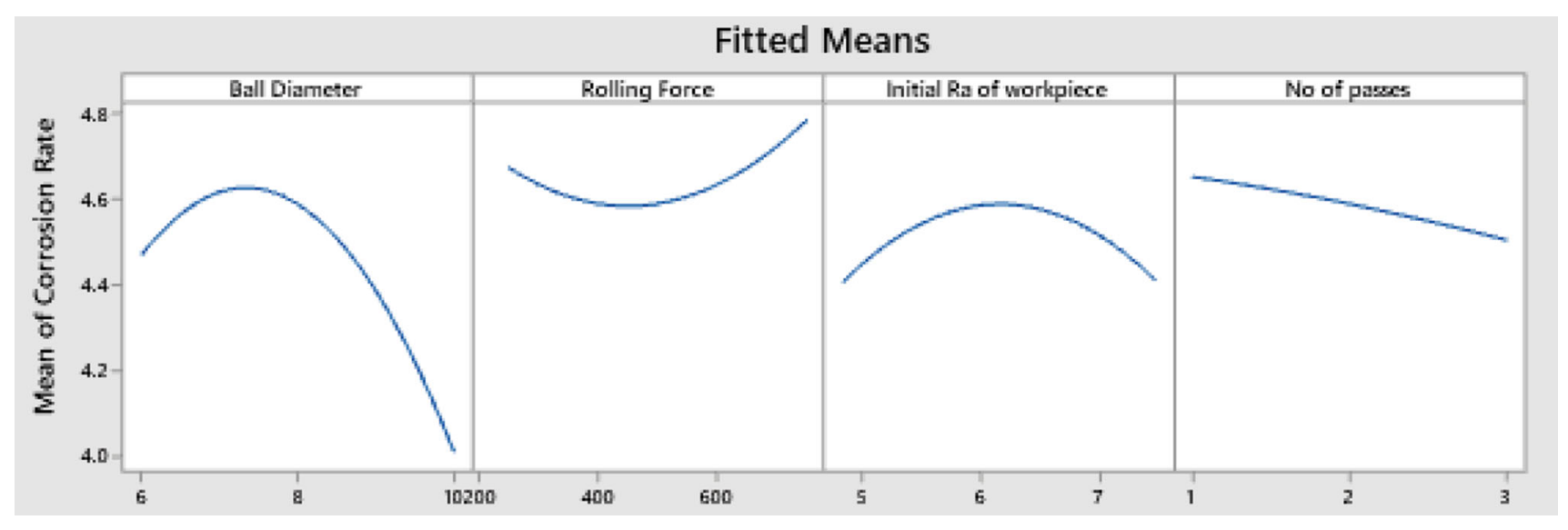

Fig. 6 Main effect plot for corrosion rate of TACDR samples in $3.5 \% \mathrm{NaCl}$ solution

\section{Fitted Means}

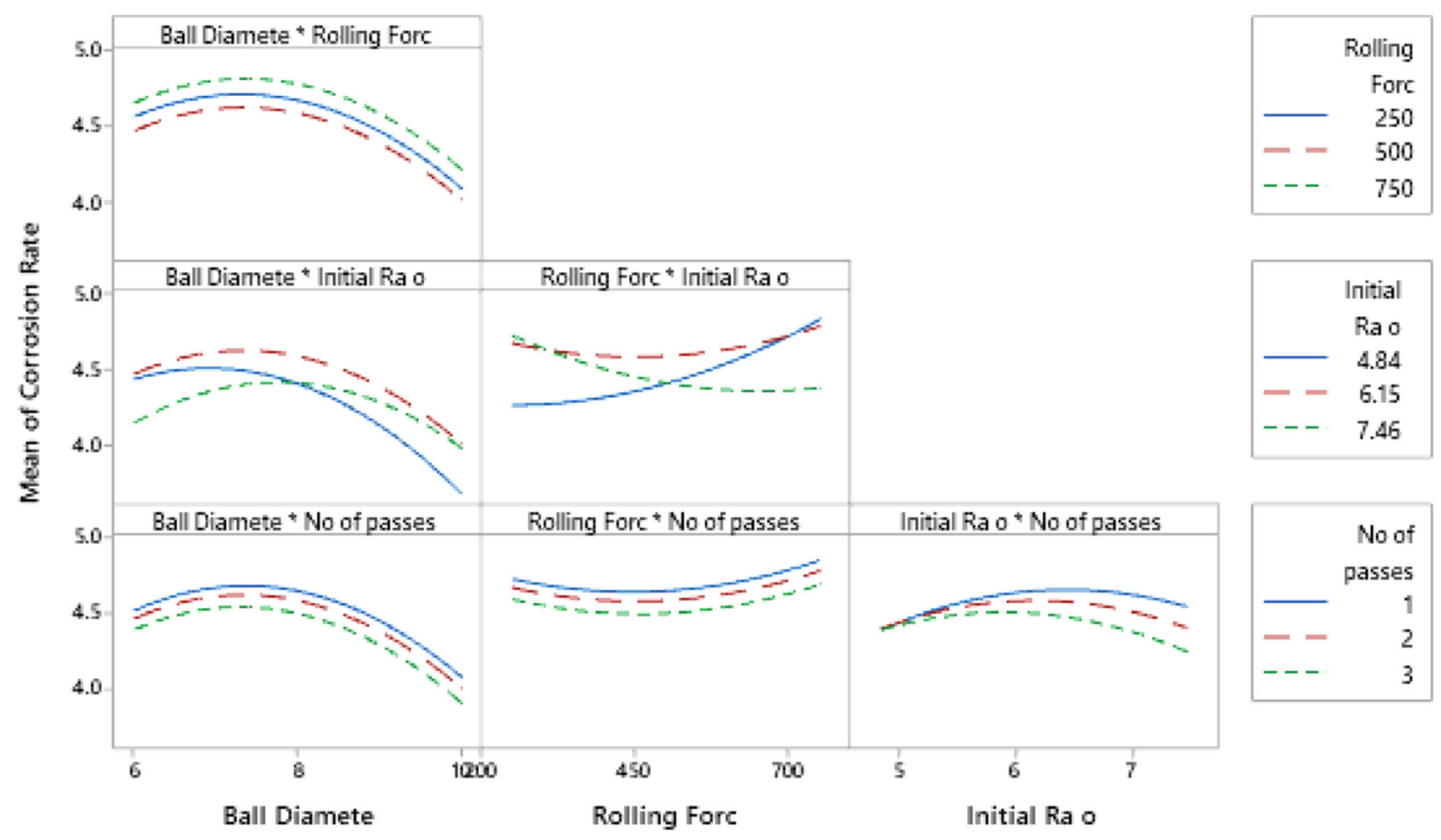

Fig. 7 Interaction effect plot for corrosion rate of TACDR samples in $3.5 \% \mathrm{NaCl}$ solution

\subsection{Corrosion Rate Measurement from Weight Loss Method}

The corrosion rate measurement is done by using the weight loss method for the TADCR specimens operated with various process parameter settings, which is summarized in Table 3. The relative effect of each process parameter on the corrosion rate is presented in the analysis of variance (ANOVA) in Table 4. The results of ANOVA indicate that ball diameter has the greatest influence on the corrosion rate with a $33 \%$ contribution followed by the interaction effect of rolling force and initial roughness of the workpiece with a $29 \%$ contribution. The rate of corrosion increases with a decrease in ball diameter, an increase in rolling force, initial roughness of the workpiece, and number of tool passes, as displayed in the main effect plot shown in Fig. 6. The "fitted means" interaction plot depends on the thought "smaller the better" for the corrosion rate. In the interaction plot, if there exists an interaction between the factors at certain levels, they are represented by crossed lines. Straight, parallel lines represent no evidence of an interaction between the factors. From Fig. 7, it is noted that significant interaction exists between the rolling force and the initial roughness of the workpiece. The parameters $S, R^{2}$, and adjusted $R^{2}$ measure how well the model fits the data. The values of $S, R^{2}$, and adjusted $R^{2}$ will assist in choosing the model with the best fit when comparing various models for the examination. The smaller value of $S$ indicates that the model better forecasts the response. 
Table 5 The values of coefficient of determination obtained from ANOVA for corrosion rate

\begin{tabular}{lccc}
\hline $\boldsymbol{S}$ & $\boldsymbol{R}^{\mathbf{2}}$ & $\boldsymbol{R}^{\mathbf{2}}$ (adj) & $\boldsymbol{R}^{\mathbf{2}}$ (pred) \\
\hline 0.0558897 & $98.61 \%$ & $97.40 \%$ & $91.88 \%$ \\
\hline
\end{tabular}

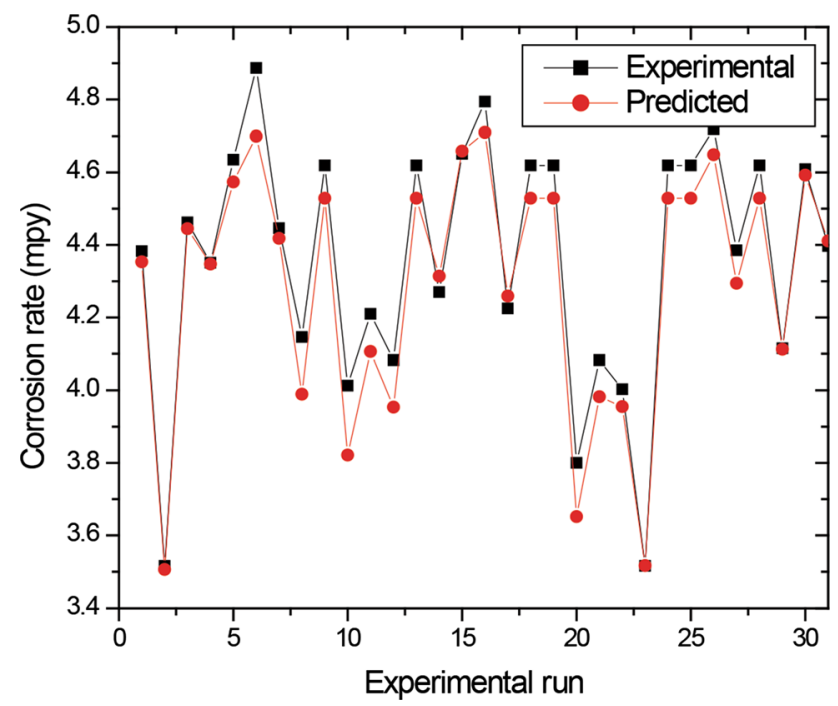

Fig. 8 Plot showing the comparison between experimental and predicted values of the corrosion rate of TADCR samples

As the values of $R^{2}$ and $R_{(\text {adj })}^{2}$ approach 1 , the model better fits the data (Ref 55). According to Table $4, R_{(\text {adj }}^{2}$ is relatively close to 1 (Table 5).

An empirical model is developed from the principles of response surface methodology for the corrosion rate. The predicted values from the empirical model are compared with the experimentally measured values. Based on the empirical model developed for the response, the contour plots are drawn to visualize the effect of process parameters. An empirical model is developed for the corrosion rate based on experimental values and is given as Eq 1. The model acquired was used to foresee the response for all values of variables inside the cutoff points considered. The model is satisfactory at a $95 \%$ certainty level. At the point when $\mathrm{P}$ values are under 0.05 , the acquired models are viewed as measurably noteworthy. It exhibits that the terms picked in the model have substantial effects on the responses:

$$
\begin{aligned}
\mathrm{CR}= & -3.545+0.928 X_{1}+0.002164 X_{2}+1.293 X_{3} \\
& +0.372 X_{4}-0.08642 X_{1}^{2}+0.000002 X_{2}^{2}-0.1040 X_{3}^{2} \\
& -0.0103 X_{4}^{2}+0.000016 X_{1} X_{2}+0.05597 X_{1} X_{3} \\
& -0.00574 X_{1} X_{4}-0.000693 X_{2} X_{3}-0.000025 X_{2} X_{4} \\
& -0.0563 X_{3} X_{4}
\end{aligned}
$$

At a $95 \%$ confidence level, a close match is observed between the predicted and experimentally obtained values, as shown in Fig. 8. It shows that the residuals fall on a straight line suggesting that the errors are distributed normally, and the maximum average error is found to be only about $1.68 \%$ between the experimental and predicted values.

From Fig. 9, it is seen that the alteration in the size of the ball influences the corrosion rate. The large contact area of the larger diameter ball enters the surface to a smaller depth than a small-diameter ball under definite rolling force. From Fig. 9, it is also revealed that by increasing the ball diameter and rolling force, the rate of corrosion decreases. The increase in the pressure of ball during the TADCR process on the workpiece surface squeezes most of the asperities and increases the flow of metal on the surface. This leads to the filling of more peaks and/ or valleys that existed on the subsurface due to turning. As the surface finish is enhanced, the area of the surface, which comes into contact with the corrosive medium, decreases, and thus decrease in corrosion rate is observed. Further increase in rolling force will diminish the surface finish because expanding the rolling force past a specific value disintegrates the surface finish on account of over solidifying and hence chipping of the surface layers. This is the reason behind increase in corrosion rate at higher rolling force. A blend of larger ball diameter with lesser rolling force, low initial roughness, and more number of tool passes will lead to a considerable reduction in corrosion rate, as shown in the response optimization plot (Fig. 10). The outcomes show that the ball diameter is one of the most critical variables influencing the corrosion rate. It tends to be seen that larger ball diameter with lower initial roughness of the workpiece resulted in a lesser corrosion rate. It is observed that the mix between more number of tool passes and larger ball diameter and lower rolling force with lower initial roughness of the workpiece brings about an extensive improvement in the corrosion rate. The optimum TADCR parameters for achieving lower corrosion rates are determined as ball diameter of $10 \mathrm{~mm}$, rolling force of $250 \mathrm{~N}$, initial roughness of $4.84 \mu \mathrm{m}$, and number of passes of 3 .

\subsection{Multiresponse Optimization of TADCR Process Variables}

The desirability function approach (DFA) is one of the most widely used methods for the optimization of multiple response processes. It is based on the idea that the "quality" of a product or process that has multiple quality characteristics, with one of them outside of some "desired" limits, is entirely unacceptable. The method finds operating conditions that provide the "most desirable" response values. Figure 11 shows the multiresponse optimization plot for surface finish and corrosion rates together obtained from the desirability function approach. It is a mathematical process to discover the optimal values of input parameters and multiple responses concurrently by using the optimum input parameter levels. Both surface finish and corrosion rates have been allotted a target value, as given in Table 6. Here, the aim is to achieve an excellent surface finish with a lower corrosion rate. In the multiresponse optimization plot, the main effect plots of surface finish and corrosion rate with desirability $(d)$ are given separately. Along with this, composite desirability (D) is also provided. The use of composite desirability is to evaluate how well the settings optimize a set of responses (surface finish and corrosion rate) overall. Desirability has a range of zero to one. One represents the ideal case; zero indicates that one or more responses are outside their acceptable limits. Composite desirability is the weighted geometric mean of the individual desirabilities for the responses. 


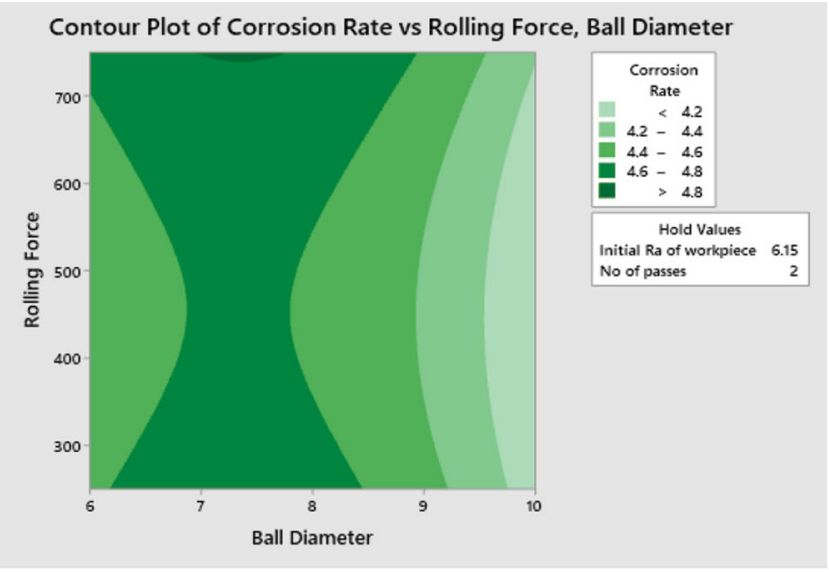

(a)

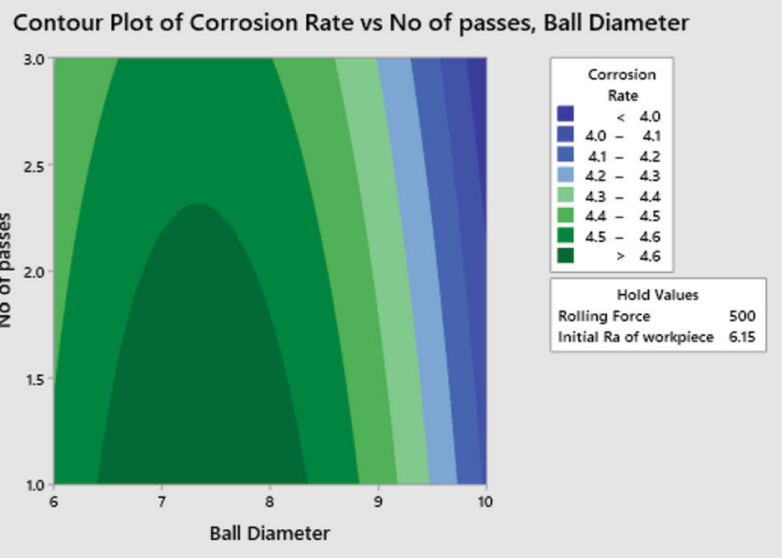

(c)

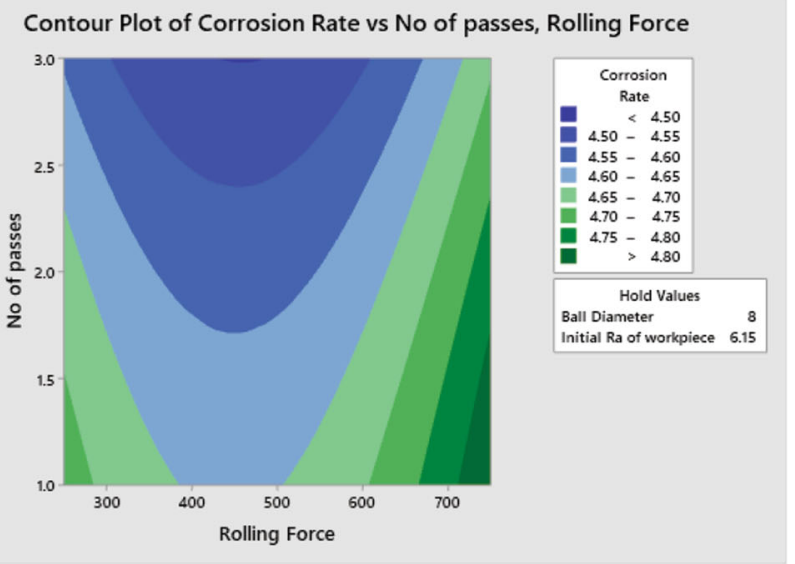

(e)

Fig. 9 Contour plot for corrosion rate of TADCR samples

Figure 11 shows the multiresponse optimization plot for surface finish and corrosion rates together. From Fig. 11, it can be found that a ball diameter of $10 \mathrm{~mm}$, a rolling force of $325.75 \mathrm{~N}$, initial roughness of $4.84 \mu \mathrm{m}$, and number of passes of 3 give better values for the two responses under consideration with composite desirability of 0.9939 .

3.5.1 Confirmation Experiments Performed at Optimum Parameter Setting. A set of validation experiments was performed at optimal parameter settings to confirm the enhancement made to performance characteristics. The purpose of
Contour Plot of Corrosion Ra vs Initial Ra of workpiece, Ball Diameter
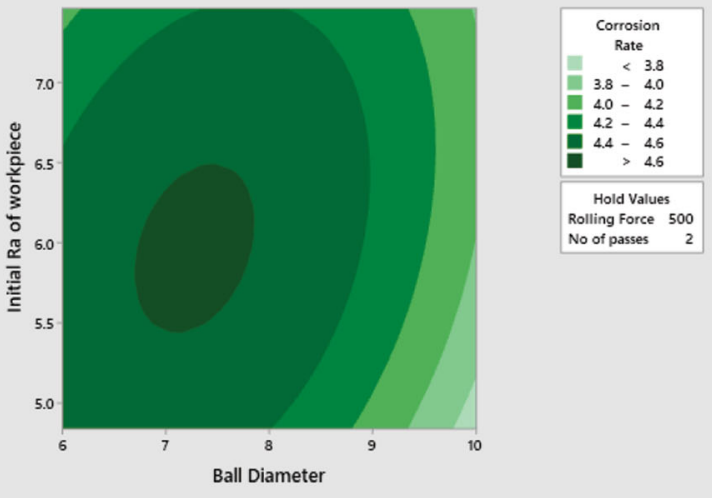

(b)

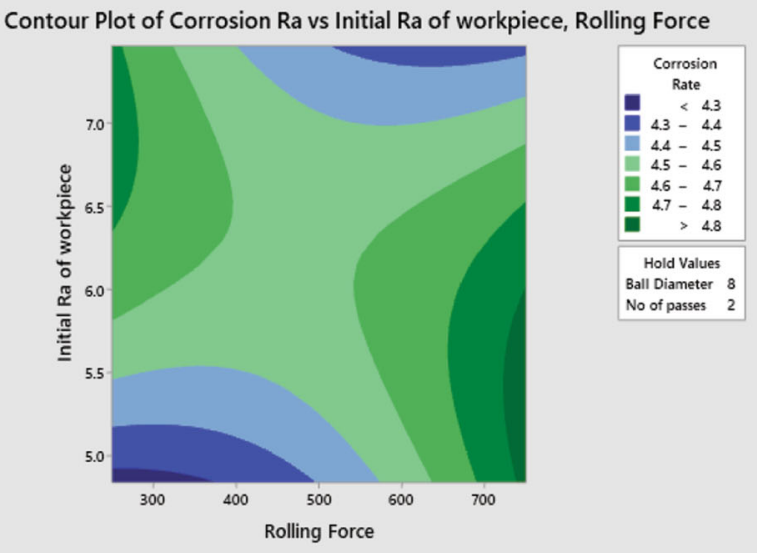

(d)

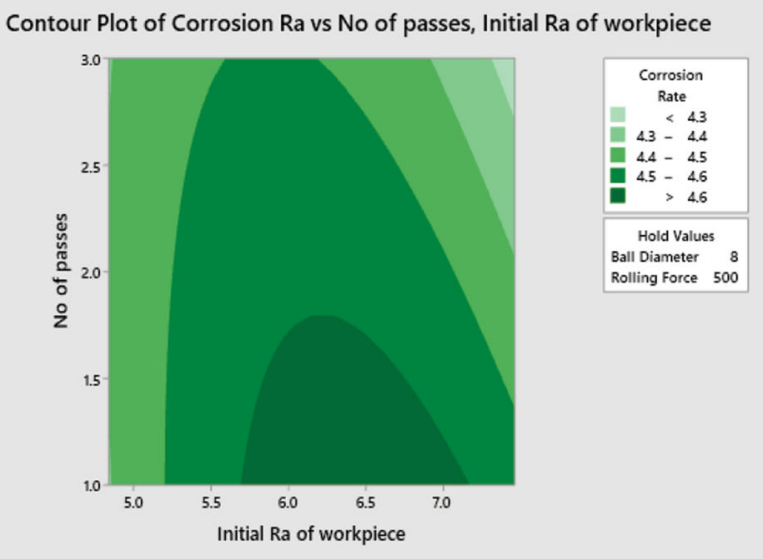

(f)

validation trials is to verify the repetitiveness of the investigated results and validate the accuracy of the predictive model. TADCR process parameters are set at optimum levels as determined from DFA and response surfaces, as depicted in Table 7.

The results from the confirmation experiments of the corrosion rate obtained at optimum process parameters are shown in Table 8. From the results, it is observed that the predicted results are in agreement with experimental test results. Based on the experimental work at the optimum parameter setting, the absolute average error between the 


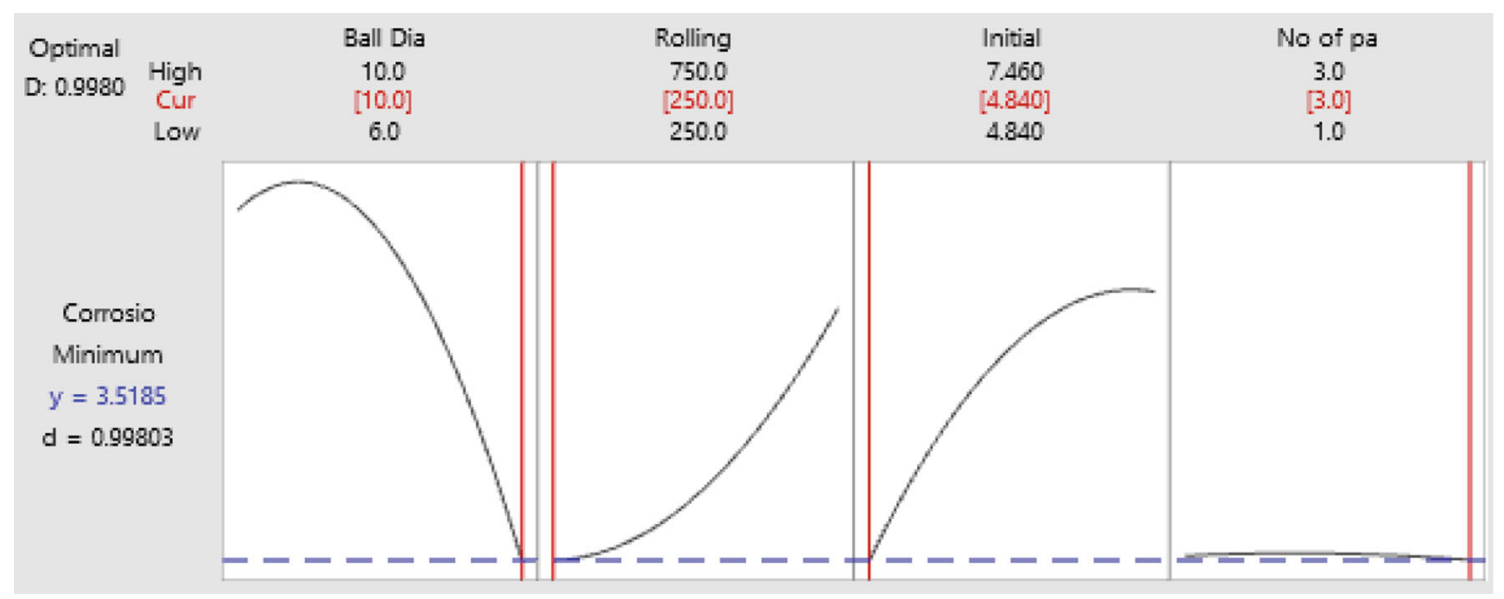

Fig. 10 Response optimization plot for corrosion rate of TADCR samples

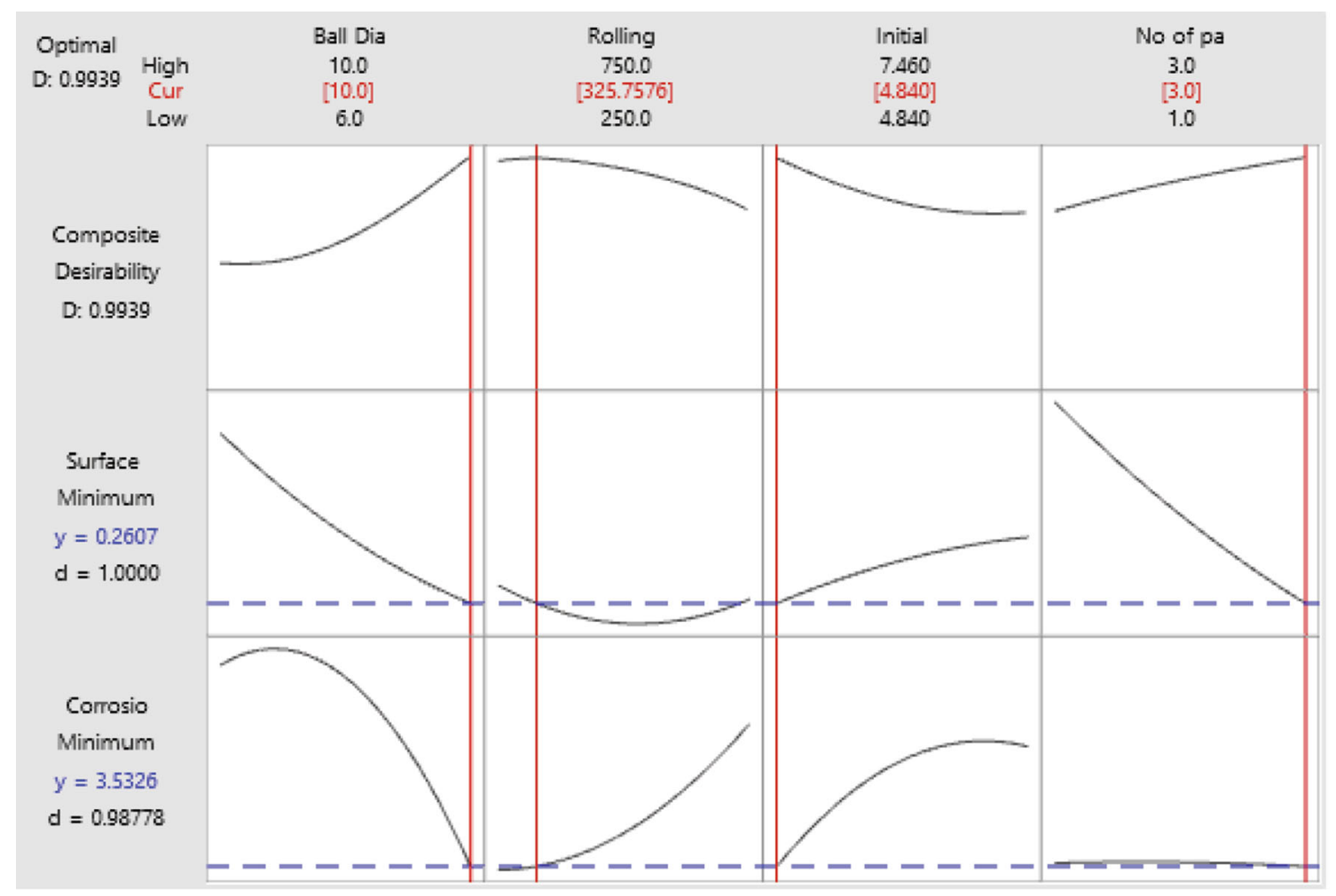

Fig. 11 Multiresponse optimization plot for surface finish and corrosion rate

Table 6 Responses and their target values for optimization

\begin{tabular}{lc}
\hline Response & Target value \\
\hline Surface finish & $0.261 \mu \mathrm{m}$ \\
Corrosion rate & $3.516 \mathrm{mpy}$ \\
\hline
\end{tabular}

Table 7 Optimum process parameters and their levels determined from DFA

\begin{tabular}{ll}
\hline Parameter & Level \\
\hline Ball diameter & $10 \mathrm{~mm}$ \\
Rolling force & $325.75 \mathrm{~N}$ \\
Initial roughness of the workpiece & $4.84 \mu \mathrm{m}$ \\
Number of passes & 3 \\
\hline
\end{tabular}


Table 8 Confirmation experiments result for corrosion rate

\begin{tabular}{|c|c|c|c|c|c|c|c|c|}
\hline \multirow[b]{2}{*}{ Parameters } & \multicolumn{4}{|c|}{ Optimum combination } & \multirow[b]{2}{*}{ Measured } & \multirow[b]{2}{*}{ Average } & \multirow[b]{2}{*}{ Predicted } & \multirow[b]{2}{*}{ Error } \\
\hline & $X_{1}$ & $X_{2}$ & $\mathbf{X}_{3}$ & $X_{4}$ & & & & \\
\hline Corrosion rate (mpy) & 10 & 325.75 & 4.84 & 3 & $\begin{array}{l}3.634 \\
3.593 \\
3.564 \\
3.715 \\
3.616\end{array}$ & 3.624 & 3.508 & $3.2 \%$ \\
\hline
\end{tabular}

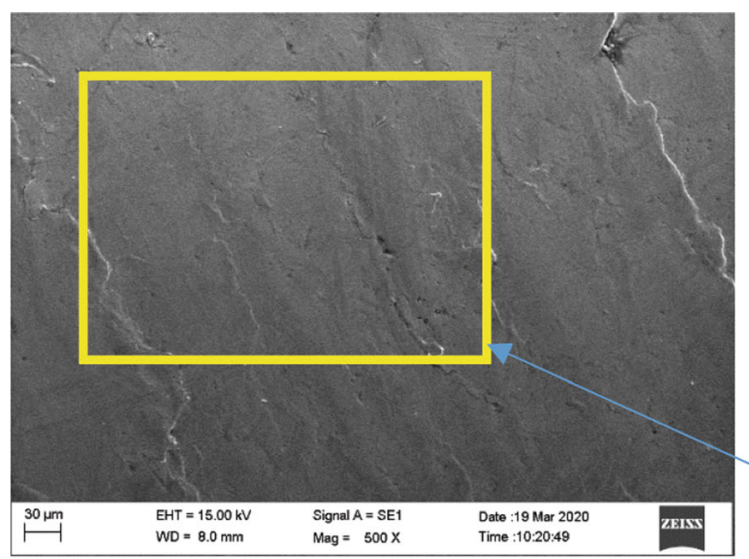

(a)

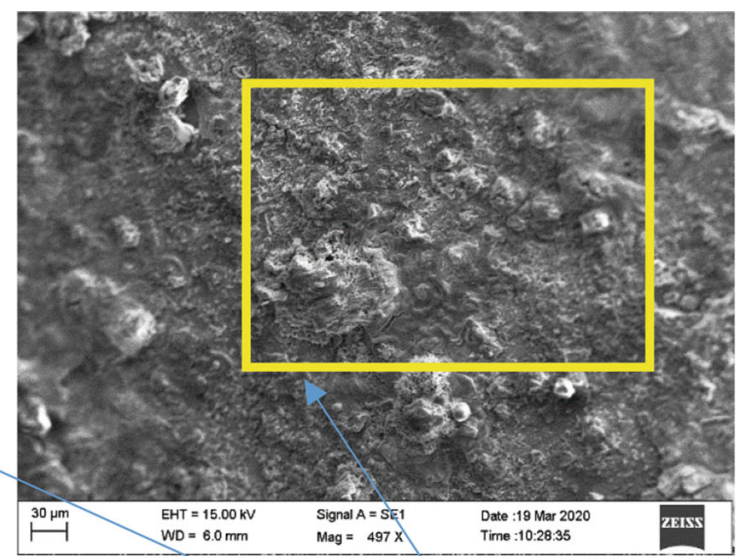

(b)

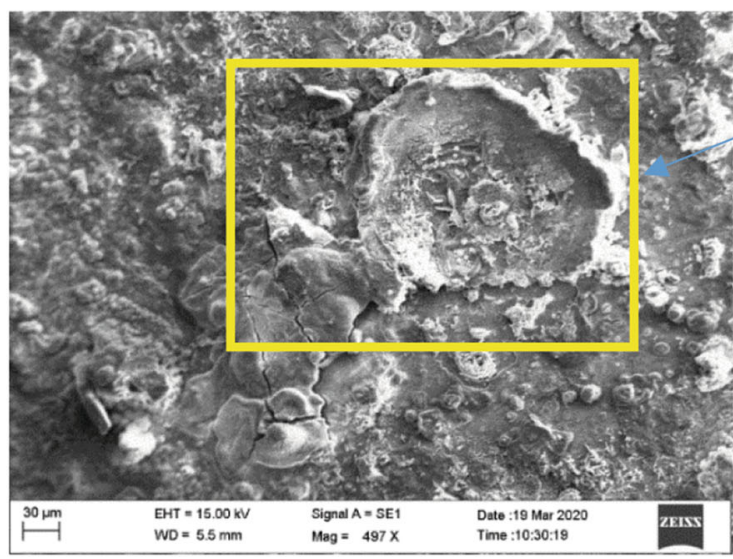

locations where EDS data collected

(c)

Fig. 12 SEM images: (a) AISI 4140 TADCR sample, (b) TADCR sample no. $6+3.5 \% \mathrm{NaCl}$, and (c) TADCR sample no. $23+3.5 \% \mathrm{NaCl}$

experimental and predicted values for the corrosion rate is calculated as $3.2 \%$.

\subsection{Surface Morphology Studies}

Once the optimum process parameters were identified for TADCR, in order to study the surface morphology, SEM and AFM analysis was carried out. For the SEM and AFM analysis, only three samples were selected (TADCR sample, TADCR sample where the lowest corrosion rate was observed, and TADCR sample where the highest corrosion rate was observed) out of the 31 samples.
3.6.1 SEM and Energy-Dispersive Spectroscopy (EDS) Analysis. SEM images of the AISI 4140 TADCR steel sample (Fig. 12a) and the TADCR samples for the experimental run order 6 and 23 (refer Table 3) where the lowest and highest corrosion rates were observed (Fig. 12b and c) during the experiments with $3.5 \% \mathrm{NaCl}$ solution as the corrosive medium immersed for a duration of $936 \mathrm{~h}$ were taken for the analysis. The TADCR sample did not display any evident flaws, excluding a few nanosize abrasions, as shown in Fig. 12(a). When the surface of deep-cold-rolled AISI 4140 steel sample was observed in higher magnification in contact 


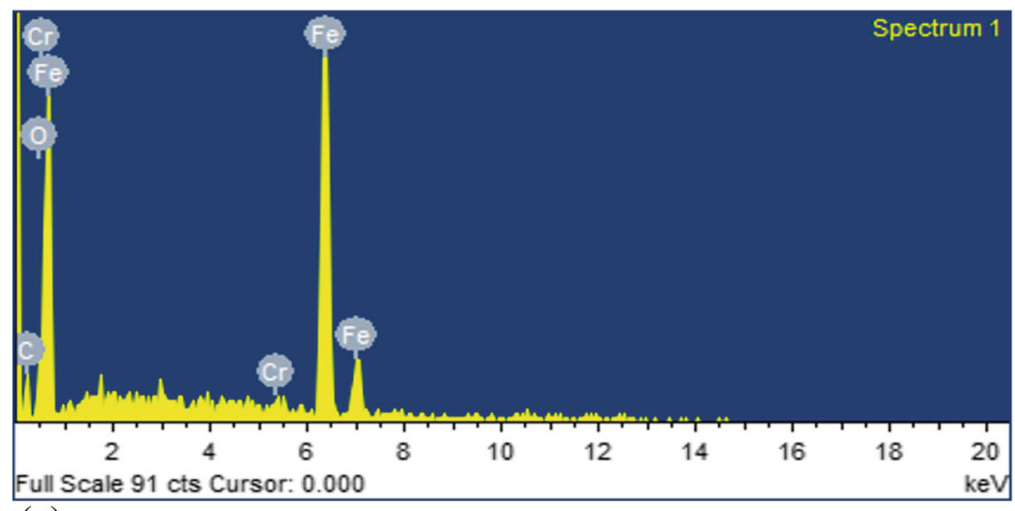

\begin{tabular}{|c|c|}
\hline Element & Weight (\%) \\
\hline $\mathbf{C}$ & 7.76 \\
\hline $\mathbf{O}$ & 2.61 \\
\hline $\mathbf{C r}$ & 1.26 \\
\hline Fe & 88.37 \\
\hline
\end{tabular}

(a)

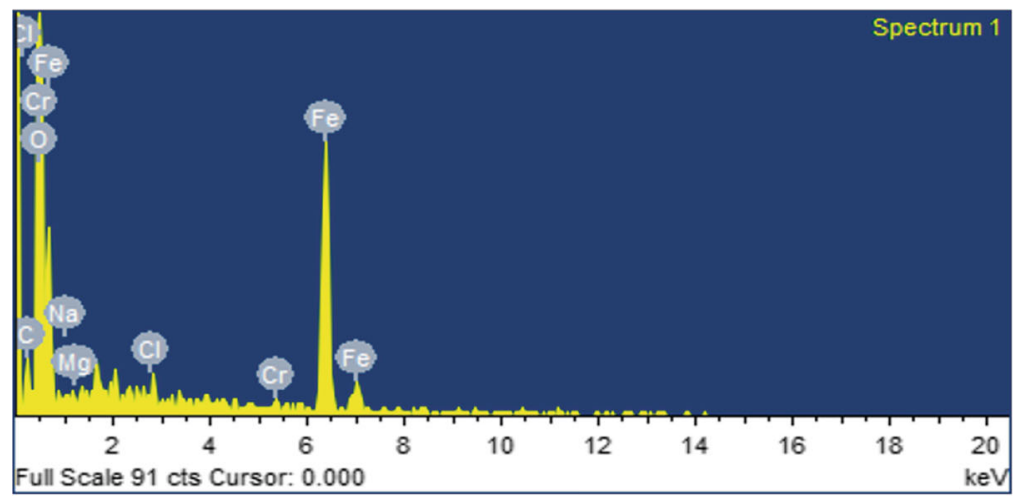

\begin{tabular}{|c|c|}
\hline Element & Weight (\%) \\
\hline \hline $\mathbf{C}$ & 12.85 \\
\hline $\mathbf{O}$ & 37.21 \\
$\mathbf{N a}$ & 0.33 \\
\hline $\mathbf{C l}$ & 0.10 \\
\hline $\mathbf{C r}$ & 0.83 \\
\hline $\mathbf{F e}$ & 48.69 \\
\hline
\end{tabular}

(b)

Fig. 13 EDS spectra: (a) AISI 4140 TADCR sample and (b) TADCR AISI 4140 steel sample $+3.5 \% \mathrm{NaCl}$

with $3.5 \% \mathrm{NaCl}$ for $936 \mathrm{~h}$, its surface seems to be rough, as shown in (b) and (c). It is detected that the surface of sample no. 23 had lesser roughness compared to sample no. 06 which is attributed to less corrosion and higher corrosion resistance. SEM analysis confirmed the corrosion and its intensity of corrosion on the metal surface.

The EDS was analyzed for the TADCR AISI 4140 steel sample before corrosion and after corrosion with a $3.5 \% \mathrm{NaCl}$ solution and is shown in Fig. 13. The data of the elemental analysis are also given in the same figure. The surface atomic composition before corrosion confirms the surface composition of the metal sample. In general, the Fe percentage decreased after immersion in $3.5 \% \mathrm{NaCl}$ solution and oxygen percentage increased. After the corrosion process, there is a presence of $\mathrm{Na}$ and $\mathrm{Cl}$ atoms in $\mathrm{EDS}$, confirming the corrosion process of metal with $\mathrm{NaCl}$ due to the dissolution of a metal layer.

3.6.2 AFM Analysis. The 3D AFM method is one of the methodologies for investigating the surface topography at the nano- and microlevels. The optical profilometry provides qualitative images at the microscale level. The AFM images of TADCR AISI 4140 steel are given in Fig. 14 for samples 6 and 23. The samples are exposed to $3.5 \% \mathrm{NaCl}$ which is taken after exposure for $936 \mathrm{~h}$, and AFM analysis was done on the surface. Surface roughness of samples 6 and 23 when in contact with $3.5 \% \mathrm{NaCl}$ has increased when compared to the TADCR sample without immersion. The increase in roughness can be attributed to the higher corrosion tendency of sample no. 6 when compared to sample no. 23. The $\mathrm{R}_{\mathrm{a}}$ values show that the roughness of the material immersed in $3.5 \% \mathrm{NaCl}$ solution for the TADCR sample no. 6 increases to $304 \mathrm{~nm}$ from $40.3 \mathrm{~nm}$ and for the TADCR sample no. 23 increases to $186 \mathrm{~nm}$ from $27 \mathrm{~nm}$. From the AFM analysis, it is also revealed that the corrosion rate is directly proportional to the $\mathrm{R}_{\mathrm{a}}$ value. AISI 4140 steel sample operated with a ball of $10 \mathrm{~mm}$ diameter and $250 \mathrm{~N}$ rolling force resulted in lower $\mathrm{R}_{\mathrm{a}}$ value when compared to the sample operated with a ball of $6 \mathrm{~mm}$ diameter and $750 \mathrm{~N}$ rolling force.

\section{Conclusions}

Stringent demands in modern manufacturing have created the need to produce parts that have a better finish, high corrosion resistance, and service life. The research is aimed at studying the influence of process variables on surface enhancement and corrosion rate of TADCR of AISI 4140 steel. DOE is used to investigate and analyze the effects systematically. Study with CCD and RSM used to evaluate the surface hardness, surface finish, and corrosion rate results in the following conclusions:

- The results from the experiment depict that compared to the untreated samples, deep cold rolling significantly enhanced the surface hardness, to a maximum $(306 \mathrm{HV})$, which then reaches the bulk hardness (225 HV) measured toward the core of the specimen. The rolling force and ball diameter play an essential role in improving the surface hardness of TADCR specimens with a contribution of 66.22 and $16.29 \%$. 

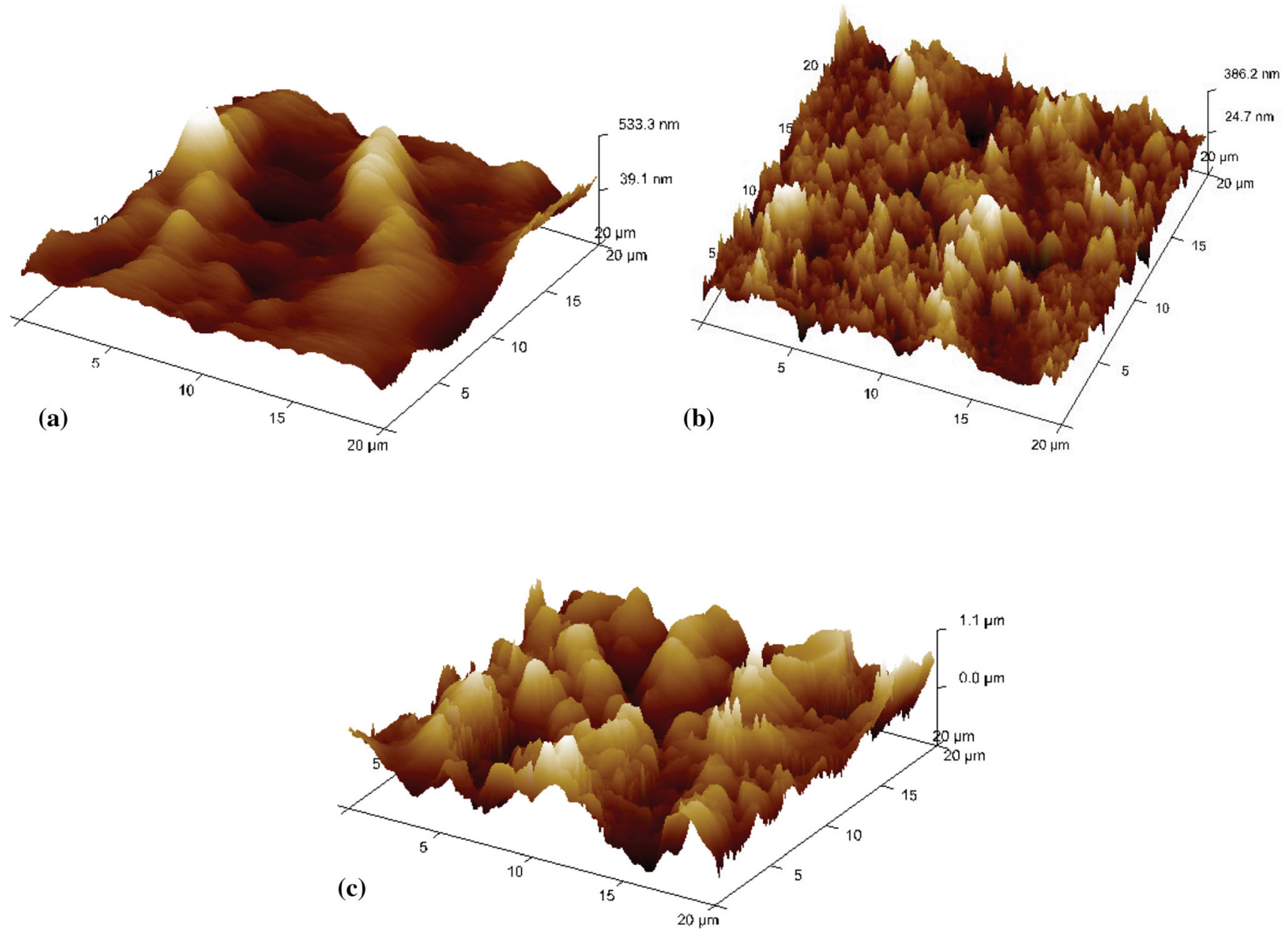

Fig. 14 AFM images: (a) AISI 4140 TADCR sample, (b) TADCR sample no. $6+3.5 \% \mathrm{NaCl}$ and (c) TADCR sample no. $23+3.5 \% \mathrm{NaCl}$

- The surface topography results show that all the experimental runs treated in various conditions enhance the surface finish when compared with the untreated samples. The number of tool passes and ball diameter are the two most significant parameters playing the primary role in enhancing the surface finish with 68.22 and $10.35 \%$ contributions respectively.

- The ball diameter plays a significant role in reducing the corrosion rate of TADCR specimens with a $33 \%$ contribution followed by the interaction effect of rolling force and initial roughness of the workpiece with a $29 \%$ contribution. The improved corrosion resistance of the TADCR sample is due to the refinement of grains, higher surface hardness, and residual compressive stress.

- The study revealed a significant compressive stress layer up to $300 \mu \mathrm{m}$ below the surface with a rolling force of $750 \mathrm{~N}$. The study also revealed one-to-one correlations between the rolling force, increase in microhardness, the magnitude of residual compressive stresses.

- An empirical model developed for the corrosion rate based on the experimental values shows a perfect fit with the experimental value.

- The optimum parameter settings determined are ball diameter of $10 \mathrm{~mm}$, rolling force of $325.75 \mathrm{~N}$, initial roughness of $4.84 \mu \mathrm{m}$, and number of tool passes of 3 . Confirmation experiments were conducted with the opti- mum parameter setting to validate the improvements in response, and it is observed that the deviation between optimum predicted and confirmatory experimental values is about $5 \%$.

- SEM and AFM analysis shows a relatively smooth surface of the AISI 4140 steel sample processed with a deep-coldrolling technique operated with a $10-\mathrm{mm}$-diameter ball and $250 \mathrm{~N}$ rolling force.

\section{Funding}

Open access funding provided by Manipal Academy of Higher Education, Manipal.

\section{Open Access}

This article is licensed under a Creative Commons Attribution 4.0 International License, which permits use, sharing, adaptation, distribution and reproduction in any medium or format, as long as you give appropriate credit to the original author(s) and the source, provide a link to the Creative Commons licence, and indicate if changes were made. The images or other third party material in this article are included in the article's Creative Commons licence, unless indicated otherwise in a credit line to the material. If material is not included in the article's Creative Commons licence 
and your intended use is not permitted by statutory regulation or exceeds the permitted use, you will need to obtain permission directly from the copyright holder. To view a copy of this licence, visit http://creativecommons.org/licenses/by/4.0/.

\section{References}

1. A.T. Bozdana, On the Mechanical Surface Enhancement Techniques in Aerospace Industry-A Review of Technology, Aircr. Eng. Aerosp. Technol., 2005, 77(4), p 279-292

2. Thomas Wegene, Thomas Niendorf, and Torben Oevermann, On the Evolution of Residual Stresses, Microstructure and Cyclic Performance of High-Manganese Austenitic Twip-Steel After Deep Rolling, Metals (Basel), 2019, 9, p 825

3. F.C. Campbell, Elements of Metallurgy and Engineering Alloys, ASM International, Cleveland, 2008

4. N. Ben Moussa, K. Gharbi, I. Chaieb, and N. Ben Fredj, Improvement of AISI, 304 Austenitic Stainless Steel Low-Cycle Fatigue Life by Initial and Intermittent Deep Rolling, Int. J. Adv. Manuf. Technol., 2019, 101(1), p 435-449

5. W. Zielecki, M. Bucior, T. Trzepiecinski, and K. Ochał, Effect of Slide Burnishing of Shoulder Fillets on the Fatigue Strength of X19NiCrMo4 Steel Shafts, Int. J. Adv. Manuf. Technol., 2020, 106(5), p 2583-2593

6. I. Altenberger, B. Scholtes, U. Martin, and H. Oettel, Cyclic Deformation and Near Surface Microstructures of Shot Peened or Deep Rolled Austenitic Stainless Steel AISI, 304, Mater. Sci. Eng., A, 1999, 264(1), p 1-16

7. S. Saalfeld, B. Scholtes, and T. Niendorf, On the Influence of Overloads on the Fatigue Performance of Deep Rolled Steel SAE 1045, Int. J. Fatigue, 2019, 126, p 221-230

8. G.O. Barbara Reggiani, Experimental Investigation on the Effect of Shot Peening and Deep Rolling on the Fatigue Response of High Strength Fasteners, Metals (Basel)., 2019, 9, p 1093

9. E. Brinksmeier, J.T. Cammett, W. König, P. Leskovar, J. Peters, and H.K. Tönshoff, Residual Stresses-Measurement and Causes in Machining Processes, CIRP Ann., 1982, 31(2), p 491-510

10. C. Liu et al., Fretting Fatigue Characteristics of Ti-6Al-4 V Alloy with a Gradient Nanostructured Surface Layer Induced by Ultrasonic Surface Rolling Process, Int. J. Fatigue, 2019, 125, p 249-260

11. W. Wang, S.N. DharmeshKumar, and Sridhar Idapalapati, Effect of Surface Mechanical Treatments on the Microstructure-Property-Performance of Engineering Alloys, Materials (Basel), 2019, 12, p 2503

12. G.D. Revankar, R. Shetty, S.S. Rao, and V.N. Gaitonde, Wear Resistance Enhancement of Titanium Alloy (Ti-6Al-4 V) by Ball Burnishing Process, J. Mater. Res. Technol., 2017, 6(1), p 13-32

13. K. Konefal, M. Korzynski, Z. Byczkowska, and K. Korzynska, Improved Corrosion Resistance of Stainless Steel X6CrNiMoTi17-12-2 by Slide Diamond Burnishing, J. Mater. Process. Technol., 2013, 213(11), p 1997-2004

14. S.M. Lee, W.G. Lee, Y.H. Kim, and H. Jang, Surface Roughness and the Corrosion Resistance of 21Cr Ferritic Stainless Steel, Corros. Sci., 2012, 63, p 404-409

15. J. Tang, H.Y. Luo, and Y.B. Zhang, Enhancing the Surface Integrity and Corrosion Resistance of Ti-6Al-4 V Titanium Alloy Through Cryogenic Burnishing, Int. J. Adv. Manuf. Technol., 2017, 88(9), p 2785-2793

16. A. Tolga Bozdana, N.N.Z. Gindy, and H. Li, Deep Cold Rolling with Ultrasonic Vibrations-A New Mechanical Surface Enhancement Technique, Int. J. Mach. Tools Manuf, 2005, 45(6), p 713-718

17. R.K. Nalla, I. Altenberger, U. Noster, G.Y. Liu, B. Scholtes, and R.O. Ritchie, On the Influence of Mechanical Surface Treatments-Deep Rolling and Laser Shock Peening - On the Fatigue Behavior of Ti6Al-4 V at Ambient and Elevated Temperatures, Mater. Sci. Eng., A, 2003, 355(1), p 216-230

18. L. Tan, D. Zhang, C. Yao, and J. Ren, Effects of Ultrasonic Surface Rolling Parameters on Surface Integrity of TC17 Alloy, J. Mater. Eng. Perform., 2019, 28(11), p 6736-6745
19. L.G.A. Fonseca and A.R. de Faria, Crankshaft Deep Rolling Analysis Through Energy Balance Simulation Output, J. Brazilian Soc. Mech. Sci. Eng., 2019, 41(10), p 430

20. M. Nakajima, K. Tokaji, H. Itoga, and H.-N. Ko, Morphology of StepWise S-N Curves Depending on Work-Hardened Layer and Humidity in A High-Strength Steel, Fatigue Fract. Eng. Mater. Struct., 2003, 26(12), p 1113-1118

21. A. Toppo, R. Kaul, M.G. Pujar, U. Kamachi Mudali, and L.M. Kukreja, Enhancement of Corrosion Resistance of Type 304 Stainless Steel Through a Novel Thermo-mechanical Surface Treatment, J. Mater. Eng. Perform., 2013, 22(2), p 632-639

22. D. Meyer and N. Wielki, Internal Reinforced Domains by Intermediate Deep Rolling in Additive Manufacturing, CIRP Ann., 2019, 68(1), p 579-582

23. X. Luo et al., Effect of Deep Surface Rolling on Microstructure and Properties of Az91 Magnesium Alloy, Trans. Nonferrous Met. Soc. China, 2019, 29(7), p 1424-1429

24. C.M. Gill, N. Fox, and P.J. Withers, Shakedown of Deep Cold Rolling Residual Stresses in Titanium Alloys, J. Phys. D Appl. Phys., 2008, 41, p $1-5$

25. K.A. Afshin Manouchehrifar, Finite Element Simulation of Deep Rolling and Evaluate the Influence of Parameters on Residual Stress, Recent Res. Appl. Mech., 2009, 1, p 121-127

26. S. Primee and P. Juijerm, Modified Mechanical Surface Treatment for Optimized Fatigue Performance of Martensitic Stainless Steel AISI, 420, Met. Mater. Int., 2019, 9, p 1-7

27. D. Kumar, S. Idapalapati, W. Wang, and A. Bhowmik, Microstructural Characteristics and Strengthening Mechanisms in A Polycrystalline NiBased Superalloy Under Deep Cold Rolling, Mater. Sci. Eng., A, 2019, 753, p 285-299

28. Y.D. Ye, X.P. Li, Z.Y. Sun, H.B. Wang, and G.Y. Tang, Enhanced Surface Mechanical Properties and Microstructure Evolution of Commercial Pure Titanium Under Electropulsing-Assisted Ultrasonic Surface Rolling Process, Acta Metall. Sin. English Lett., 2018, 31(12), p $1272-1280$

29. D. Mombeini, and A. Atrian (2018) Investigation of Deep Cold Rolling Effects on the Bending Fatigue of Brass C38500. Lat. Am. J. Solids Struct. 15(4)

30. P.J.P. Donhongprai, Optimized Fatigue Performance of Martensitic Stainless Steel AISI, 440C Using Deep Rolling Integrated Into Hardening Process, J. Min. Metall. Sect. B Metall., 2018, 54(1), p 67-71

31. S. Saalfeld, T. Oevermann, T. Niendorf, and B. Scholtes, Consequences of Deep Rolling on the Fatigue Behavior of Steel SAE 1045 at High Loading Amplitudes, Int. J. Fatigue, 2019, 118, p 192-201

32. S.M.K. Raghavendra Prabhu, S.S. Sharma, K. Jagannath, and Krishna Kumar, Surface Improvement of Shafts by Turn-Assisted Deep Cold Rolling Process, MATEC Web Conf., 2016, 77, p 1-4

33. N. Tsuji, S. Tanaka, and T. Takasugi, Evaluation of Surface-Modified Ti-6Al-4 V alloy by combination of plasma-carburizing and deeprolling, Mater. Sci. Eng., A, 2008, 488(1), p 139-145

34. Y.-F. Jia et al., Enhanced Surface Strengthening of Titanium Treated by Combined Surface Deep-Rolling and Oxygen Boost Diffusion Technique, Corros. Sci., 2019, 157, p 256-267

35. P.R. Prabhu, D. Prabhu, and P. Rao, Optimization of the parameters influencing the inhibition efficiency of $6063 \mathrm{Al}$ alloy in $0.5 \mathrm{M} \mathrm{NaOH}$ using response surface methodology, Int. J. Mech. Prod. Eng. Res. Dev., 2020, 10(2), p 51-64

36. D.C. Montgomery, Design and Analysis of Experiments: Response Surface Method and Designs, Wiley, New Jersey, 2005

37. P. R. Prabhu, D. Prabhu, and P. Rao (2020) Analysis of Garcinia Indica Choisy Extract as Eco-Friendly Corrosion Inhibitor for Aluminum in Phosphoric Acid Using the Design of Experiment. J. Mater. Res. Technol.

38. A. Sagbas, Analysis and Optimization of Surface Roughness in the Ball Burnishing Process using Response Surface Methodology and Desirabilty Function, Adv. Eng. Softw., 2011, 42(11), p 992-998

39. A.M. Hassan and A.M. Maqableh, The Effects of Initial Burnishing Parameters on Non-Ferrous Components, J. Mater. Process. Technol., 2000, 102(1), p 115-121

40. B. Sachin, S. Narendranath, and D. Chakradhar, Application of Desirability Approach to Optimize the Control Factors in Cryogenic Diamond Burnishing, Arab. J. Sci. Eng., 2020, 45(2), p 1305-1317 
41. R. Teimouri, S. Amini, and A.B. Bami, Evaluation of Optimized Surface Properties and Residual Stress in Ultrasonic Assisted Ball Burnishing of AA6061-T6, Measurement, 2018 , 116, p 129-139

42. P.J. Ross, Taguchi Techniques for Quality Engineering: Loss Function, Orthogonal Experiments, Parameter and Tolerance Design, McGrawHill, New York, 1996

43. T.-T. Nguyen, L.-H. Cao, X.-P. Dang, T.-A. Nguyen, and Q.-H. Trinh, Multi-Objective Optimization of the Flat Burnishing Process for Energy Efficiency and Surface Characteristics, Mater. Manuf. Process., 2019, 34(16), p 1888-1901

44. Y.X. Zhao, Fatigue Crack Growth Law Covering Threshold and Fracture Toughness for Railway LZ50 Axle Steel, Adv. Mater. Res., 2012, 544, p 280-285

45. C. Dureau et al., On the Influence of Ultrasonic Surface Mechanical Attrition Treatment (SMAT) on the Fatigue Behavior of the 304L Austenitic Stainless Steel, Metals (Basel), 2020, 10(1), p 100

46. S. Ren et al., Enhanced Surface Properties and Microstructure Evolution of $\mathrm{Cr} 12 \mathrm{MoV}$ Using Ultrasonic Surface Rolling Process Combined with Deep Cryogenic Treatment, J. Mater. Eng. Perform., 2019, 28(2), p 1132-1140

47. W. Zhao et al., Improving the Fretting and Corrosion Fatigue Performance of $300 \mathrm{~m}$ Ultra-High Strength Steel Using the Ultrasonic Surface Rolling Process, Int. J. Fatigue, 2019, 121, p 30-38

48. Z.-M. Wang, Y.-F. Jia, X.-C. Zhang, Y. Fu, C.-C. Zhang, and S.-T. Tu, Effects of Different Mechanical Surface Enhancement Techniques on
Surface Integrity and Fatigue Properties of Ti-6Al-4 V: A Review, Crit. Rev. Solid State Mater. Sci., 2019, 44(6), p 445-469

49. D. Meyer and J. Kämmler, Surface Integrity of AISI, 4140 After Deep Rolling with Varied External and Internal Loads, Procedia CIRP, 2016, 45, p 363-366

50. D.B. Nataliya Lyubenova, Finite Element Modelling and Investigation of the Process Parameters in Deep Rolling of AISI, 4140 Steel, $J$. Mater. Sci. Eng. B, 2015, 5(7), p 277-287

51. H.C. Hao Fu, Yan Liu, Xu Qian, Xiulin Yan, and Guiying Yang, Effect of Deep Rolling Parameters on Surface Integrity of LZ50 Axles, Int. J. Mod. Phys. B, 2019, 33(25), p 1-12

52. E. Oberg and R.E. Green, Machinery's Handbook: A Reference Book for the Mechanical Engineer, Designer, Manufacturing Engineer, Toolmaker, and Machinist, Industrial Press, Draftsman, 1996

53. G.M.C.P.R. Prabhu, S.S. Sharma, and S.M. Kulkarni, Turn-Assisted Deep Cold Rolling: An Innovative Manufacturing Method for the improvement of Fatigue Life, Int. J. Appl. Eng. Res., 2015, 10(78), p 64-68

54. A. G1-90, Standard Practice for Preparation, Cleaning, and Evaluating Corrosion Test Specimens. 1999

55. I. State College, PA: Minitab, Minitab 17 Statistical Software. 2019

Publisher's Note Springer Nature remains neutral with regard to jurisdictional claims in published maps and institutional affiliations. 